\title{
Association of Circulating Vascular Endothelial Growth Factor Levels With Autoimmune Diseases: A Systematic Review and Meta- Analysis
}

OPEN ACCESS

Edited by: Antonio Serrano,

Research Institute Hospital 12 de Octubre, Spain

Reviewed by: Wang-Dong $X u$, Southwest Medical University, China Shi-Yang Guan, Anhui Medical University, China

*Correspondence: Yongzhe Li yongzhelipumch@126.com

${ }^{t}$ These authors have contributed equally to this work and share first authorship

Specialty section: This article was submitted to Autoimmune and Autoinflammatory Disorders, a section of the journal

Frontiers in Immunology

Received: 01 March 2021 Accepted: 27 April 2021 Published: 27 May 2021

Citation:

Zhan H, Li H, Liu C, Cheng L, Yan S and Li Y (2021) Association of Circulating Vascular Endothelial Growth Factor Levels With Autoimmune Diseases: A Systematic Review and Meta-Analysis.

Front. Immunol. 12:674343. doi: 10.3389/fimmu.2021.674343

\author{
Haoting Zhan ${ }^{1,2 \dagger}$, Haolong $\mathrm{Li}^{1,2 \dagger}$, Chenxi Liu ${ }^{1,2}$, Linlin Cheng ${ }^{1,2}$, Songxin Yan ${ }^{1,2}$ \\ and Yongzhe $\mathrm{Li}^{1,2 *}$
}

1 Department of Clinical Laboratory, Peking Union Medical College Hospital, Peking Union Medical College and Chinese Academy of Medical Sciences, Beijing, China, 2 Department, State Key Laboratory of Complex, Severe and Rare Diseases, Peking Union Medical College Hospital, Chinese Academy of Medical Science and Peking Union Medical College, Beijing, China

Background: Autoimmune diseases (ADs) are characterized by immune-mediated tissue damage, in which angiogenesis is a prominent pathogenic mechanism. Vascular endothelial growth factor (VEGF), an angiogenesis modulator, is significantly elevated in several ADs including rheumatoid arthritis (RA), systemic sclerosis (SSc), and systemic lupus erythematosus (SLE). We determined whether circulating VEGF levels were associated with ADs based on pooled evidence.

Methods: The analyses included 165 studies from the PubMed, EMBASE, Cochrane Library, and Web of Science databases and fulfilled the study criteria. Comparisons of circulating VEGF levels between patients with ADs and healthy controls were performed by determining pooled standard mean differences (SMDs) with 95\% confidence intervals (Cls) in a random-effect model using STATA 16.0. Subgroup, sensitivity, and metaregression analyses were performed to determine heterogeneity and to test robustness.

Results: Compared with healthy subjects, circulating VEGF levels were significantly higher in patients with SLE (SMD 0.84, 95\% Cl 0.25-1.44, $P=0.0056$ ), RA (SMD 1.48, 95\% Cl 0.82-2.15, $P<0.0001$ ), SSc (SMD 0.56, 95\% Cl 0.36-0.75, $P<0.0001$ ), Behcet's disease (SMD 1.65, 95\% Cl 0.88-2.41, $P<0.0001$ ), Kawasaki disease (SMD 2.41, 95\% Cl 0.10-4.72, $\mathrm{P}=0.0406$ ), ankylosing spondylitis (SMD 0.78, 95\% Cl 0.23-1.33, $\mathrm{P}=$ 0.0052), inflammatory bowel disease (SMD 0.57, 95\% Cl 0.43-0.71, P <0.0001), psoriasis (SMD 0.98, 95\% Cl 0.62-1.34, P <0.0001), and Graves' disease (SMD 0.69, 95\% Cl 0.20-1.19, P = 0.0056). Circulating VEGF levels correlated with disease activity and hematological parameters in ADs.

Conclusion: Circulating VEGF levels were associated with ADs and could predict disease manifestations, severity and activity in patients with ADs. 


\section{Systematic Review Registration: PROSPERO, identifier CRD42021227843.}

Keywords: diagnosis, disease activity, autoimmune disease, vascular endothelial growth factor, angiogenesis

\section{INTRODUCTION}

Angiogenesis, a hallmark of inflammatory activation, is an integral part of pathogenic processes including endothelial cell proliferation and migration and subsequent neoangiogenesis and remodeling in autoimmune diseases (ADs). Synovial pannus initiates the invasion of cartilage and subchondral bone to perpetuate rheumatoid arthritis (RA) $(1,2)$, whereas ankylosing spondylitis (AS) is characterized by increased vascularity and vascular lesions (3). Vascular endothelial dysfunction and injury are considered as the primum movens triggering Kawasaki disease (KD), systemic lupus erythematosus (SLE), inflammatory bowel disease (IBD), Behcet's disease (BD), systemic sclerosis (SSc), and psoriasis (PsA) (4-9). Therefore, early detection of vascular involvement is pivotal in $\mathrm{AD}$ diagnosis.

Vascular endothelial growth factor (VEGF)-A, generally known as VEGF, is a crucial regulator of endothelial dysfunction, capillary permeability, and angiogenesis. For example, serum VEGF level and intrathyroid microvessel density were reported to be increased patients with Graves' disease (GD) compared to healthy control (HC) subjects (10). Increased serum VEGF and significant difference in diffused and limited SSc suggest VEGF as a potential surrogate indicator of capillary damage (11). Strong VEGF expression in synovial fluid and serum of patients with RA was shown to lead to synovial neovascularization and destruction in cartilage and bones (12, 13). VEGF was reported to be overexpressed in the skin and peripheral blood of patients with PsA (14). Serum VEGF levels were shown to be elevated and to correlate with disease activity and severity in PsA, SLE, BD, IBD, KD, and AS (14-19). These findings suggest VEGF as a potential pathogenic factor with promising diagnostic value in ADs. However, no clinical guidelines currently recommend serum VEGF evaluation in routine care and counseling of patients with ADs, and intensive studies are warranted to identify the clinical implications of the findings regarding VEGF's role in ADs to date and to resolve contradictory results (20-24).

Given the inconsistency among these findings and lower statistic power of the studies, we performed a systematic review and meta-analysis to generate independent results and recognize the source of heterogeneity. In the present study, we aimed to determine whether circulating VEGF was a causative factor in ADs.

\section{MATERIALS AND METHODS}

\section{Literature Search}

The present systematic review with meta-analysis was performed according to the PRISMA guidelines (PROSPERO registration number, CRD42021227843). Two authors (HTZ and HLL) independently searched the PubMed, Embase, Cochrane Library, and the Web of Science databases for studies published until October 14. The detailed search strategies are provided in the online Supplemental Materials. Reference lists were manually retrieved.

\section{Eligibility Criteria}

Without restrictions on time, language, ethnicity, and geographical region, studies satisfying the following criteria were included: (1) case-control or cohort studies on the association between circulating VEGF and ADs including SLE, RA, SSc, BD, KD, AS, IBD, PsA, and GD; (2) HCs without ADs (2); available data on circulating VEGF levels (serum or plasma); (3) sufficient data on VEGF levels for both HCs and patients with ADs to evaluate standard mean differences (SMDs) with $95 \%$ confidence intervals (CIs). Studies based on animal and cellular models, those comprising HCs with insufficient data; and editorial letters with insufficient data were excluded.

\section{Data Extraction and Quality Assessment}

Two independent investigators (HTZ and HLL) individually screened the literature and extracted and evaluated the data. Any discrepancies were resolved by consensus or by a third opinion (YZL). Study number, name of the first author, publication year, country, study type, sample type, inclusion and exclusion criteria, demographic features, aggregated number of subjects and circulating VEGF levels in patients with $\mathrm{ADs}$ and HCs, diagnostic criteria, type of VEGF assay, and treatment history and strategy were extracted into pre-designed charts. For meta-analysis, continuous variables were translated from medians (interquartile range [IQR] or range) to means \pm standard deviation (25). Newcastle-Ottawa quality assessment scale was used to evaluate study quality. Further details of the pooled studies were obtained by directly contacting the authors if warranted.

\section{Data Analysis}

STATA V.16.0 was used to perform the meta-analysis. SMDs with 95\% CIs were used to estimate the pooled results and compare circulating VEGF levels between patients and HC groups. Random-effect model was used for analysis. Significant heterogeneity was ascertained based on a $p$ value of $\leq 0.10$ using the Cochrane $Q$ test or an $\mathrm{I}^{2}$ value of $>50 \%$. Subgroup, sensitivity, and meta-regression analyses were performed to identify the source of heterogeneity and to test robustness. Spearman correlation coefficients were transformed into Pearson's $\mathrm{r}$ values, which were converted to Fisher's $\mathrm{z}$ values to obtain approximately normal distributions. Ultimately, the summary Fisher's $\mathrm{z}$ values were converted into summary $\mathrm{r}$ values. Summary $\mathrm{r}$ values of $0.8-1.0,0.6-0.8,0.4-0.6$, and $0.2-0.4$ 
indicated extreme, high, and moderate relevance and poor correlation, respectively (details provided in the online Supplemental Materials). Publication bias was assessed by Egger's linear regression test and contour-enhanced funnel plots with collaborative meta-trim. A two-sided $P<0.05$ was considered to indicate statistical significance.

\section{RESULTS}

\section{Search Results and Population Characteristics}

The literature search is summarized in Figure 1. After removing duplicate studies $(\mathrm{n}=3,322)$ and irrelevant publications $(\mathrm{n}=$ 8,673), 298 articles were analyzed and the full texts of 273 articles were read. Thirty-two full-text articles were eliminated due to incomplete data or unrelated outcomes. Among 241 eligible studies meeting the inclusion criteria, 76 articles were excluded due to unextractable data, insufficient data on HCs, irrelevant VEGF sample type (urine/synovial fluid/tear fluid), or inappropriate disease control groups. Finally, 165 studies were included in the meta-analysis, with $28,29,40,13,8,12,16,23$, and six studies on SLE $(20,21,26-51)$, RA $(12,22-24,38,43,52-$ $74)$, SSc $(11,38,39,64,75-110)$, BD (111-123), KD $(18,124-$ 130), AS (55, 73, 131-140), IBD (141-156), PsA (12, 14, 135, 136, 157-175) and GD (10, 176-180), respectively. The main study characteristics are summarized in Table 1 and Appendix 1. The studies were medium-to-high quality based on the NewcastleOttawa quality assessment scale scores (range, 4-9).

\section{Meta-Analysis of the Association Between Circulating VEGF and SLE}

Circulating VEGF levels were significantly higher in SLE than in HC (SMD 0.84, 95\%CI 0.25-1.44, $P=0.0056$ ) (Figure 2A). Additionally, circulating VEGF was higher in active SLE than in inactive SLE (SMD 0.80, 95\%CI 0.02-1.59, $P=0.0454$ ) (Figure 2B-i), serum VEGF levels remained remarkable higher in active

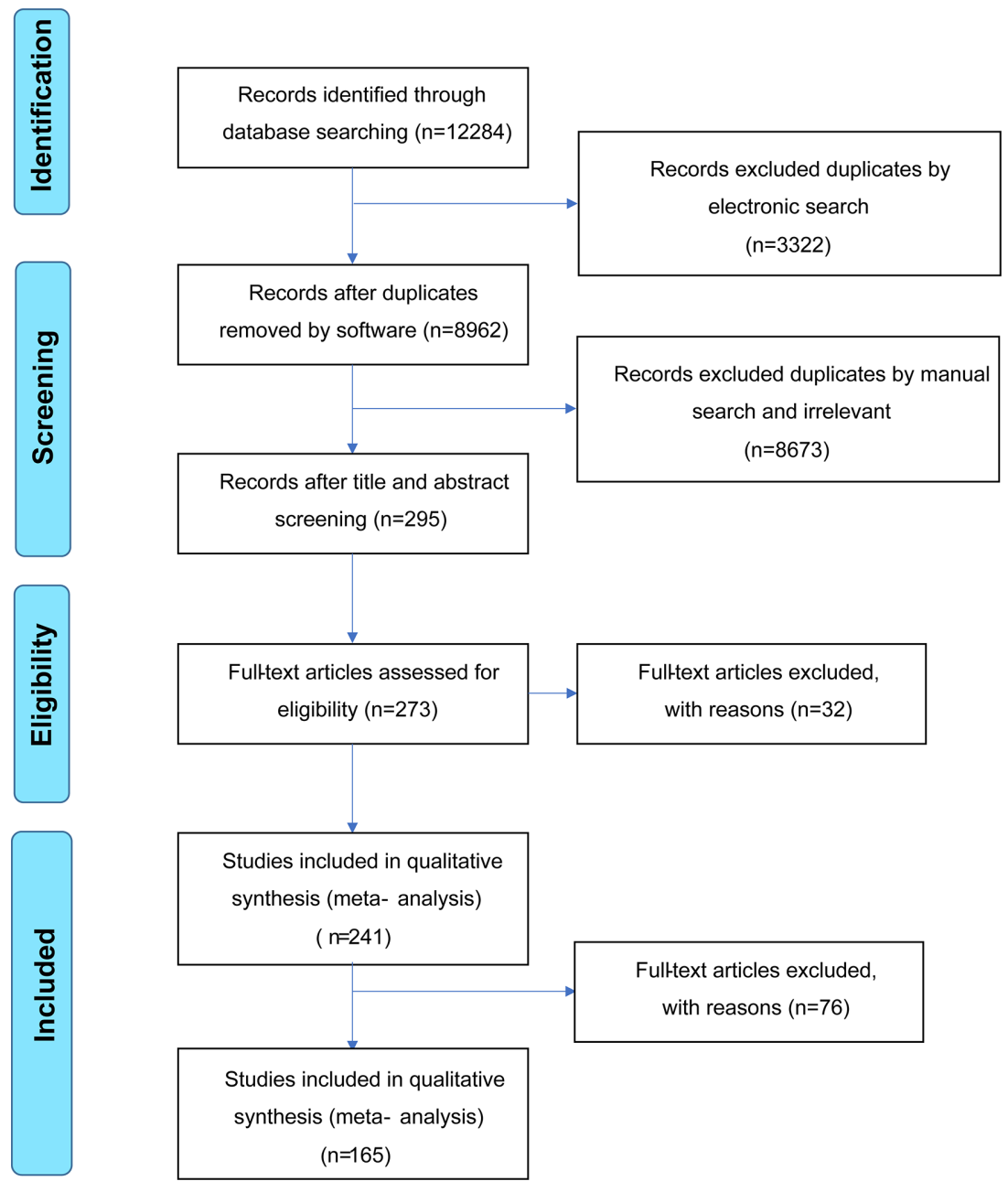

FIGURE 1 | Flow diagram of included/excluded studies. 
TABLE 1 | Population characteristics of the studies included in the meta-analysis.

\begin{tabular}{|c|c|c|c|c|c|c|c|c|c|}
\hline \multirow[t]{2}{*}{ Year } & \multirow[t]{2}{*}{ Author } & \multirow[t]{2}{*}{ Country } & \multirow[t]{2}{*}{ Study type } & \multicolumn{3}{|c|}{ SLE } & \multicolumn{3}{|c|}{$\mathrm{HC}$} \\
\hline & & & & Sample size & Female (\%) & Age (years) & $\begin{array}{l}\text { Sample } \\
\text { size }\end{array}$ & $\begin{array}{c}\text { Female } \\
(\%)\end{array}$ & Age (years) \\
\hline 2015 & Barbulescu AL (20) & Romania & case-control & 18 & $16(88.88)$ & $45.00 \pm 10.81$ & 17 & $16(94.11)$ & range: 19-64 \\
\hline 2019 & Barraclough M (21) & UK & case-control & 36 & $34(94)$ & $40 \pm 12.41$ & 30 & $30(100)$ & $32 \pm 14.44$ \\
\hline 2008 & Ciprandi G (26) & Italy & case-control & 40 & $40(100)$ & $41.95 \pm 8.3$ & 40 & $33(82.5)$ & $43 \pm 8.2$ \\
\hline 2009 & Colombo BM (27) & Italy & case-control & 80 & $80(100)$ & $42.6 \pm 9.1$ & 80 & $80(100)$ & $40.1 \pm 9.5$ \\
\hline 2014 & De Jesus GR (28) & Brazil & case-control & 54 & $54(100)$ & & 34 & $34(100)$ & \\
\hline 2015 & Ding $Y(29)$ & China & case-control & 41 & $30(73.2)$ & $11.1 \pm 2.4$ & 10 & & \\
\hline 2009 & Elhelaly NS (30) & Egypt & case-control & 23 & 21 (91.3) & Range 8-18 & 25 & & \\
\hline 2012 & Edelbauer M (31) & Austria & case-control & 23 & $17(73.9)$ & $15 \pm 5$ & 20 & $5(25)$ & $12 \pm 3$ \\
\hline 2018 & El-Gazzar II (32) & Egypt & case-control & 84 & $84(100)$ & $29.03 \pm 5.4$ & 33 & & \\
\hline 2017 & Ghazali WSW (33) & Malaysia & case-control & $\begin{array}{l}92 \\
\text { LN } 46 \\
\text { Non-LN } 46\end{array}$ & $\begin{array}{c}44(96) \\
46(100)\end{array}$ & $\begin{array}{c}28.48 \pm 9.93 \\
32.39 \pm 11.46\end{array}$ & 26 & $26(100)$ & $33.19 \pm 10.3$ \\
\hline 2007 & Heshmat NM (34) & Egypt & case-control & 25 & $24(96)$ & $14.1 \pm 2.6$ & 30 & $29(96.7)$ & $14.0 \pm 2.5$ \\
\hline 2009 & Hrycek A (35) & Poland & case-control & 48 & $48(100)$ & $47 \pm 14$ & 24 & $24(100)$ & $51 \pm 15$ \\
\hline 2009 & Hrycek A (36) & Poland & case-control & 21 & $21(100)$ & $51 \pm 12.4$ & 24 & $24(100)$ & $51 \pm 15.3$ \\
\hline 2008 & Ibrahim FF (37) & Egypt & case-control & 30 & $30(100)$ & $25 \pm 7.75$ & 10 & $10(100)$ & $32 \pm 7.5$ \\
\hline 1998 & Kikuchi K (38) & Japan & case-control & 17 & $14(82.4)$ & $47 \pm 12.75$ & 20 & $16(80 \%)$ & $50 \pm 12.5$ \\
\hline 2013 & Koca SS (39) & Turkey & case-control & 23 & $21(91.3)$ & $37.9 \pm 9.3$ & 28 & 22 (78.6\%) & $42.5 \pm 13.9$ \\
\hline 2007 & Kuryliszyn-Moskal A (40) & Poland & case-control & 47 & 44 (93.6) & $40.8 \pm 13.6$ & 30 & & \\
\hline 2014 & Liu J (41) & China & case-control & 75 & $59(78.7)$ & $35.42 \pm 11.79$ & 40 & $31(77.5)$ & $33.62 \pm 10.21$ \\
\hline 2018 & Merayo-Chalico J (42) & Mexico & case-control & $\begin{array}{l}\text { active SLE } 6 \\
\text { remission SLE } \\
6\end{array}$ & $\begin{array}{l}6(100) \\
6(100)\end{array}$ & $\begin{array}{l}34.6 \pm 4.2 \\
34.1 \pm 4.8\end{array}$ & 6 & $6(100)$ & $36 \pm 4.1$ \\
\hline 2016 & Novikov A (43) & Russia & case-control & 80 & $72(90)$ & $31.5 \pm 36.3$ & 28 & & \\
\hline 2012 & Moneib HA (44) & Egypt & case-control & 30 & $21(70)$ & $28.9 \pm 10.2$ & 15 & $10(66)$ & $35.00 \pm 9.48$ \\
\hline 2002 & Navarro C (45) & Mexico & case-control & 28 & $24(85.7)$ & $36.6 \pm 16.1$ & 24 & $19(79.2)$ & $29.2 \pm 8.5$ \\
\hline 2005 & Robak E (46) & Poland & case-control & 41 & $38(92.7)$ & $40.5 \pm 13.5$ & 20 & & \\
\hline 2003 & Robak E (47) & Poland & case-control & 60 & $55(91.7)$ & $41 \pm 14.25$ & 20 & $17(85)$ & $45 \pm 5.75$ \\
\hline 2013 & Robak E (48) & Poland & case-control & 60 & 56 (93.3) & $39.2 \pm 11.25$ & 20 & $17(85)$ & \\
\hline 2002 & Robak E (49) & Poland & case-control & 52 & 48 (92.3) & $41 \pm 14.75$ & 20 & $18(90)$ & $38 \pm 11.75$ \\
\hline 2017 & Willis R (50) & America & $\begin{array}{l}\text { case-control } \\
\text { cohort1 } \\
\text { cohort2 }\end{array}$ & $\begin{array}{l}312 \\
267 \\
45\end{array}$ & $\begin{array}{c}252(94.4) \\
44(97.8)\end{array}$ & $\begin{array}{l}47.6 \pm 12.4 \\
44.0 \pm 12.1\end{array}$ & 30 & 83.3 & $43.5 \pm 12.5$ \\
\hline 2014 & Zhou L (51) & China & case-control & 54 & $50(92.6)$ & $36.81 \pm 12.52$ & 28 & $22(78.6)$ & $37.82 \pm 12.86$ \\
\hline \multirow[t]{2}{*}{ Year } & Author & Country & Study type & & RA & & & $\mathrm{HC}$ & \\
\hline & & & & Sample size & Female (\%) & Age (years) & $\begin{array}{l}\text { Sample } \\
\text { size }\end{array}$ & $\begin{array}{c}\text { Female } \\
(\%)\end{array}$ & Age (years) \\
\hline 2004 & Ardicoglu $\bigcirc$ (52) & & case-conrol & 38 & & & 40 & & \\
\hline 2001 & Ballara S (12) & UK & cohort & $\begin{array}{l}\text { early } 44 \\
\text { longstanding } \\
78\end{array}$ & $\begin{array}{l}61 \\
85\end{array}$ & $\begin{array}{l}61 \pm 17.78 \\
61 \pm 14.07\end{array}$ & 31 & 65 & $49 \pm 12.59$ \\
\hline 2000 & Bottomley MJ (53) & UK & case-conrol & 61 & 51 (83.6) & $59 \pm 11.75$ & 29 & $20(69.0)$ & $34 \pm 8$ \\
\hline 2005 & Kim HR (62) & Korea & case-conrol & 30 & $24(80)$ & $50 \pm 8$ & 20 & $16(80)$ & $30 \pm 8$ \\
\hline 2016 & Deveci K (55) & Turkey & case-control & 30 & & $\begin{array}{c}\text { mean age of } \\
30-50\end{array}$ & 30 & & $\begin{array}{c}\text { mean age of } \\
30-50\end{array}$ \\
\hline 2002 & Drouart M (56) & France & case-control & 50 & $32(64)$ & $59.8 \pm 12.8$ & 64 & 30 (46.9) & $42.1 \pm 10.1$ \\
\hline 2016 & do Prado AD (57) & Brazil & case-control & 64 & $50(78.1)$ & $55.3 \pm 9.8$ & 30 & $23(76.7)$ & $55.9 \pm 11.1$ \\
\hline 2009 & Foster W (22) & UK & case-control & 66 & $41(62.1)$ & $58 \pm 14$ & 49 & $34(69.4)$ & $54 \pm 10$ \\
\hline 2018 & Gumus A (58) & Turkey & case-control & $\begin{array}{l}59 \\
\text { joint swelling } \\
(+) 31 \\
\text { joint swelling } \\
(-) 28\end{array}$ & $\begin{array}{l}27(87.10) \\
25(89.28)\end{array}$ & $\begin{array}{c}45.06 \pm 9.66 \\
45.10 \pm 13.03\end{array}$ & 25 & $20(80.0)$ & $46.4 \pm 13.3$ \\
\hline 2014 & Heard BJ (59) & Canada & case-control & 100 & & $46.5 \pm 14.5$ & 100 & & $40.0+9.5$ \\
\hline 2008 & Hetland ML (60) & Denmark & case-control & 10 & & & 10 & & \\
\hline 2003 & Hashimoto N (61) & Japan & case-control & active RA22 & $18(81.8)$ & $54 \pm 12.75$ & 11 & & \\
\hline
\end{tabular}


TABLE 1 | Continued

\begin{tabular}{|c|c|c|c|c|c|c|c|c|c|}
\hline \multirow[t]{2}{*}{ Year } & \multirow[t]{2}{*}{ Author } & \multirow[t]{2}{*}{ Country } & \multirow[t]{2}{*}{ Study type } & \multicolumn{3}{|c|}{ RA } & \multicolumn{3}{|c|}{$\mathrm{HC}$} \\
\hline & & & & Sample size & Female (\%) & Age (years) & $\begin{array}{l}\text { Sample } \\
\text { size }\end{array}$ & $\begin{array}{c}\text { Female } \\
(\%)\end{array}$ & Age (years) \\
\hline 1998 & Kikuchi K (38) & Japan & case-control & 11 & $10(90.9)$ & $51 \pm 10.75$ & 20 & $16(80)$ & $50 \pm 12.5$ \\
\hline 2007 & Cho ML (54) & Korea & case-control & 72 & & $49.6 \pm 1.3$ & 31 & & $47.1 \pm 2.1$ \\
\hline 2006 & Kuryliszyn-Moskal A (63) & Poland & case-control & 64 & $54(84.4)$ & $58.6 \pm 12.6$ & 32 & & \\
\hline 2004 & Kuwana M (64) & Japan & case-control & 11 & $11(100)$ & $59.1 \pm 12.0$ & 11 & $11(100)$ & $52.7 \pm 10.6$ \\
\hline 2010 & Milman N (65) & Canada & case-control & 47 & 78.70 & $54.3 \pm 14.25$ & & & \\
\hline 2018 & Misra S (23) & India & case-control & 50 & $46(92)$ & $35.90 \pm 18.607$ & 30 & 28 (93.3) & $34.03 \pm 10.3$ \\
\hline 2016 & Novikov A (43) & Russia & case-control & 74 & $59(79.7)$ & $54.0 \pm 13.33$ & & & \\
\hline 2001 & Olszewski WL (66) & Poland & case-control & 20 & $16(80)$ & $42 \pm 7.5$ & 20 & & $25 \pm 1$ \\
\hline 2012 & Oranskiy SP (67) & Russia & case-control & $\begin{array}{l}39 \text { (BMl } \\
\text { normal) }\end{array}$ & 82.0 & $53.0 \pm 2.75$ & 20 & 80.0 & $52.0 \pm 2.5$ \\
\hline 2010 & Ozgonenel L (68) & Turkey & case-control & 40 & $32(80)$ & $46 \pm 12.59$ & 38 & $18(47.4)$ & $44 \pm 11.11$ \\
\hline 2009 & Young HR (69) & America & case-control & 169 & 69.20 & $54.2 \pm 11.8$ & 92 & 63 & $53.2 \pm 11.6$ \\
\hline 2016 & Rodriguez-Carrio J (70) & Spain & case-control & 212 & $175(82.5)$ & $54 \pm 17.25$ & 175 & $102(58.3)$ & $51 \pm 14.25$ \\
\hline 2016 & Smets P (71) & France & case-control & 80:RA13 & $8(61.5)$ & $71 \pm 7.97$ & 37 & $24(64.9)$ & $73.35 \pm 8.55$ \\
\hline 2004 & Strunk J (72) & Germany & case-control & active RA 21 & $16(76.2)$ & range: 38-79 & 12 & $6(50)$ & range: $17-58$ \\
\hline 2010 & Tseng JC (73) & China & case-control & 50 & & & 50 & & \\
\hline 2001 & Sone H (24) & Japan & case-control & 155 & $130(83.9)$ & $57.9 \pm 12.0$ & 75 & $62(82.7)$ & $55.8 \pm 15.4$ \\
\hline 2007 & Zayed A (74) & Egypt & case-control & 40 & & range:21-57 & 20 & & \\
\hline \multirow[t]{2}{*}{ Year } & \multirow[t]{2}{*}{ Author } & \multirow[t]{2}{*}{ Country } & \multirow[t]{2}{*}{ Study type } & \multicolumn{3}{|c|}{ SSc } & \multicolumn{3}{|c|}{$\mathrm{HC}$} \\
\hline & & & & Sample size & Female (\%) & Age (years) & $\begin{array}{l}\text { Sample } \\
\text { size }\end{array}$ & $\begin{array}{c}\text { Female } \\
(\%)\end{array}$ & Age (years) \\
\hline 2018 & Alekperov R (75) & Russia & case-control & 46 & & & 20 & & \\
\hline 2004 & Allanore Y (76) & France & case-control & 40 & $33(82.5)$ & $57 \pm 12$ & 20 & $17(85)$ & $51 \pm 7$ \\
\hline 2013 & Aydogdu E (77) & Turkey & case-control & 40 & $38(95)$ & $48.35 \pm 13.2$ & 20 & $19(95)$ & $49.3 \pm 8.5$ \\
\hline 2017 & Benyamine A (78) & France & case-control & 45 & $44(97.8)$ & $61.49 \pm 11.95$ & 41 & $38(92.7)$ & $56.09 \pm 7.82$ \\
\hline 2014 & Bosello SL (79) & Italy & case-control & 28 & & & 11 & & \\
\hline 2014 & Bosello SL (80) & Italy & case-control & 24 & & & 10 & & \\
\hline 2002 & Choi JJ (11) & Korea & case-control & 48 & 45 (81.8) & $40.6 \pm 13$ & 55 & 30 & $38 \pm 6$ \\
\hline \multirow[t]{2}{*}{2017} & Chora I (81) & Italy & case-control & 55 & $49(89.0)$ & $64 \pm 11$ & 55 & $51(92.7)$ & $52 \pm 10.25$ \\
\hline & & & & VEDOSS 25 & $21(84.0)$ & $50 \pm 14.5$ & & & \\
\hline \multirow[t]{4}{*}{2016} & Cossu M (82) & Italy & case-control & UCTD/SSC 47 & $52.7 \pm 14.2$ & & 43 & & \\
\hline & & & & $\begin{array}{l}\text { SSc without } \\
\text { skin fibrisis } 48\end{array}$ & $62 \pm 13.2$ & & & & \\
\hline & & & & limited 51 & $62.1 \pm 10.4$ & & & & \\
\hline & & & & diffused 35 & $54.6 \pm 12.6$ & & & & \\
\hline 2013 & De Lauretis A (83) & UK & case-control & 74 & $59(79.7)$ & $51.4 \pm 12.1$ & 20 & $7(35)$ & $32.7 \pm 6.3$ \\
\hline 2017 & Delle Sedie A (84) & Italy & case-control & 41 & $40(97.6)$ & $56 \pm 15$ & 31 & 25 (80.6) & $50 \pm 16$ \\
\hline 2011 & Distler JHW (85) & Germany & case-control & 40 & $34(85)$ & $46 \pm 14.5$ & 66 & $44(66.7)$ & $39 \pm 13.75$ \\
\hline 2002 & Distler O (86) & Italy & case-control & 43 & 35 (81.4) & $61 \pm 13.75$ & 21 & 16 (76.2) & $55 \pm 16.75$ \\
\hline 2012 & Dunne JV (87) & Canada & case-control & 40 & $35(87.5)$ & & 40 & & \\
\hline & & & & diffused 14 & $45.5 \pm 9.5$ & & & & \\
\hline & & & & limited 26 & $\begin{array}{c}53.8 \pm \\
13.25\end{array}$ & & & & \\
\hline \multirow[t]{3}{*}{2005} & Dziankowska-Bartkowiak B & Poland & case-control & 34 & $26(76.5)$ & $48 \pm 13.5$ & 20 & $19(95.0)$ & $46 \pm 9.75$ \\
\hline & & & & diffused 15 & 8 (53.3) & $45 \pm 12$ & & & \\
\hline & & & & limited 19 & $18(94.7)$ & $50 \pm 10.75$ & & & \\
\hline \multirow[t]{3}{*}{2006} & Dziankowska-Bartkowiak B & Poland & case-control & 28 & $22(78.6)$ & $47.5 \pm 13$ & 20 & $15(75)$ & $46 \pm 9.75$ \\
\hline & & & & diffused 12 & 7 (58.3) & $48 \pm 11.5$ & & & \\
\hline & & & & limited 16 & $15(93.8)$ & $47 \pm 10.75$ & & & \\
\hline 2013 & Farouk HM (90) & Egypt & case-control & 25 & $21(84)$ & $40.3 \pm 5.86$ & 20 & $17(85)$ & $38.9 \pm 3.8$ \\
\hline 2014 & $\begin{array}{l}\text { Gkodkowska-Mrowka E } \\
(91)\end{array}$ & Poland & case-control & 66 & $60(90)$ & $53 \pm 13.25$ & 21 & $18(85.7)$ & $52 \pm 10.25$ \\
\hline 2018 & Gigante A (92) & Italy & case-control & 15 & $15(100)$ & $41 \pm 10.835$ & 10 & & $39 \pm 10.484$ \\
\hline
\end{tabular}


TABLE 1 | Continued

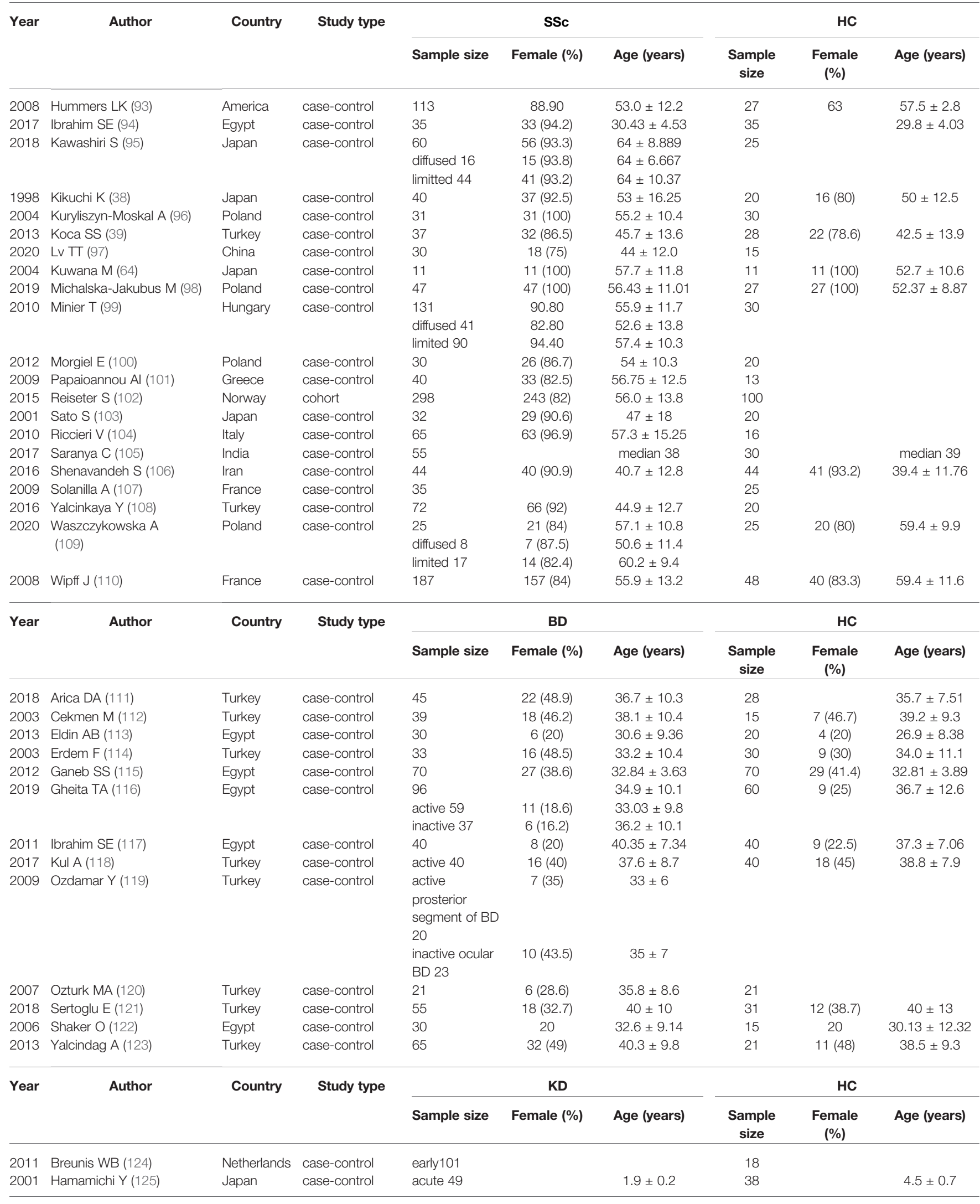


TABLE 1 | Continued

\begin{tabular}{|c|c|c|c|c|c|c|c|c|c|}
\hline \multirow[t]{2}{*}{ Year } & \multirow[t]{2}{*}{ Author } & \multirow[t]{2}{*}{ Country } & \multirow[t]{2}{*}{ Study type } & \multicolumn{3}{|c|}{ KD } & \multicolumn{3}{|c|}{$\mathrm{HC}$} \\
\hline & & & & Sample size & Female (\%) & Age (years) & $\begin{array}{l}\text { Sample } \\
\text { size }\end{array}$ & $\begin{array}{c}\text { Female } \\
(\%)\end{array}$ & Age (years) \\
\hline & & & & convalesent 30 & & $4.8 \pm 0.7$ & & & \\
\hline \multirow[t]{4}{*}{1998} & Maeno N (126) & Japan & case-control & 22 & $10(45.5)$ & $2.2 \pm 1.425$ & healthy 19 & $9(47.7)$ & $1.4 \pm 1.4$ \\
\hline & & & & acute 20 & $10(50)$ & $1.5 \pm 1.15$ & febrile 22 & $10(45.5)$ & $1.3 \pm 1.4$ \\
\hline & & & & subacute 13 & 5 (38.5) & $2.5 \pm 1.325$ & & & \\
\hline & & & & convalesent 15 & $8(53.3)$ & $1.9 \pm 1.4$ & & & \\
\hline 1999 & Ohno T (18) & Japan & case-control & $\begin{array}{l}\text { acute } 66 \\
\text { acute phase31 } \\
\text { convalescent } \\
\text { phase31 }\end{array}$ & $24(36.4)$ & $1.79 \pm 2.375$ & $\begin{array}{c}\text { healthy } 18 \\
\text { febrile } 18\end{array}$ & $\begin{array}{c}8(44.4) \\
9(50)\end{array}$ & $\begin{array}{c}4.25 \pm 1.75 \\
3.375 \pm 2.29\end{array}$ \\
\hline 2002 & Takuro Ohno (127) & Japan & case-control & $\begin{array}{l}\text { acute phase } \\
41 \\
\text { convalescent } \\
\text { phase } 41\end{array}$ & $14(34.1)$ & $1.83 \pm 2.17$ & 25 & $8(32)$ & $9 \pm 1.75$ \\
\hline 2019 & Su Y (128) & China & case-control & 90 & $51(56.7)$ & $2.55 \pm 1.72$ & $\begin{array}{l}\text { healthy } 60 \\
\text { febrile } 40\end{array}$ & $\begin{array}{c}28(46.7) \\
20(50)\end{array}$ & $\begin{array}{l}2.19 \pm 2.22 \\
2.84 \pm 1.63\end{array}$ \\
\hline 2009 & Ueno K (129) & Japan & case-control & 80 & $37(46.25)$ & $2.1 \pm 1.8$ & febrile 26 & $10(38.5)$ & $1.9 \pm 1.1$ \\
\hline 2016 & Zeng H (130) & China & case-control & 52 & & & & & \\
\hline \multirow[t]{2}{*}{ Year } & Author & Country & Study type & \multicolumn{3}{|c|}{ AS } & \multicolumn{3}{|c|}{$\mathrm{HC}$} \\
\hline & & & & Sample size & Female (\%) & Age (years) & $\begin{array}{l}\text { Sample } \\
\text { size }\end{array}$ & $\begin{array}{c}\text { Female } \\
(\%)\end{array}$ & Age (years) \\
\hline 2016 & Akar S (13)] & Turkey & case-control & 98 & & $27.7 \pm 8.6$ & 49 & & \\
\hline 2016 & Deveci K (55) & Turkey & case-control & 30 & & $\begin{array}{l}\text { mean age of } 30- \\
50\end{array}$ & 30 & & $\begin{array}{c}\text { mean age of } 30- \\
50\end{array}$ \\
\hline 2002 & Goldberger C (132) & Austria & case-control & 16 & $2(12.5)$ & $50.4 \pm 2.7$ & 8 & & \\
\hline 2015 & $\operatorname{Lin} \Pi(133)$ & China & case-control & 140 & $102(72.9)$ & $31.8 \pm 9.3$ & 90 & $72(80)$ & $30.2 \pm 8.2$ \\
\hline 2016 & Przepiera-Bedzak H (134) & Poland & case-control & 80 & $16(20)$ & $50.9 \pm 12.8$ & 21 & $8(38.1)$ & $48.2 \pm 13.5$ \\
\hline 2015 & Przepiera-Bedzak H (135) & Poland & case-control & 61 & $12(19.7)$ & $43.3 \pm 13.2$ & 29 & $19(65.5)$ & $48.2 \pm 13.5$ \\
\hline 2016 & Przepiera-Bedzak H (136) & Poland & case-control & 81 & $20(24.7)$ & $44.7 \pm 13.2$ & 30 & $19(63.3)$ & $43.5 \pm 9.4$ \\
\hline 2016 & Sakellariou GT (137) & Greece & case-control & 57 & $4(7.0)$ & $39.1 \pm 1.4$ & 34 & $2(6.0)$ & $38.8 \pm 1.0$ \\
\hline 2015 & Solmaz D (138) & Turkey & case-control & 98 & $21(21.4)$ & $39.3 \pm 10.0$ & 49 & $12(24.5)$ & $39.0 \pm 5.9$ \\
\hline 2018 & Solmaz D (139) & Turkey & case-control & 97 & $21(21.6)$ & $38 \pm 10.4$ & 48 & $12(25)$ & $41 \pm 5.0$ \\
\hline 2019 & Torres L (140) & Sweden & case-control & 204 & $87(43)$ & $49 \pm 15.56$ & 80 & & \\
\hline 2010 & Tseng JC (73) & China & case-control & 50 & & & 50 & & \\
\hline \multirow[t]{2}{*}{ Year } & \multirow[t]{2}{*}{ Author } & \multirow[t]{2}{*}{ Country } & \multirow[t]{2}{*}{ Study type } & \multicolumn{3}{|c|}{ IBD } & \multicolumn{3}{|c|}{$\mathrm{HC}$} \\
\hline & & & & Sample size & Female (\%) & Age (years) & $\begin{array}{l}\text { Sample } \\
\text { size }\end{array}$ & $\begin{array}{c}\text { Female } \\
(\%)\end{array}$ & Age (years) \\
\hline 2018 & Aksoy EK (141) & Turkey & case-control & UC 39 & $15(38.5)$ & $46.1 \pm 12.6$ & 15 & $7(46.7)$ & $41.4 \pm 12.6$ \\
\hline 2014 & Algaba A (142) & Spain & case-control & $37(\cup C=6)$ & $20(54)$ & $36 \pm 13$ & 40 & $24(60)$ & $43 \pm 9$ \\
\hline 2004 & Di Sabatino A (143) & Italy & case-control & CD 25 & & $37.8 \pm 11.25$ & 22 & & $38.3 \pm 11.25$ \\
\hline 2007 & Dueñas Pousa I (144) & Spain & case-control & CD 30 & $15(50)$ & $44 \pm 14$ & 30 & $15(50)$ & $43 \pm 14$ \\
\hline 2006 & Ferrante M (145) & Belgium & cohort & 824 & 466 (56.6) & $38.9 \pm 12.07$ & 271 & $156(57.6)$ & $28 \pm 10.37$ \\
\hline \multirow[t]{3}{*}{1999} & Griga T (146) & Germany & case-control & 27 & & & 10 & $5(50)$ & $29.3 \pm 6.1$ \\
\hline & & & & CD 19 & $8(42.1)$ & $34.8 \pm 11.0$ & & & \\
\hline & & & & UC 8 & $3(37.5)$ & $46.6 \pm 19.5$ & & & \\
\hline \multirow[t]{3}{*}{1998} & Griga T (147) & Germany & case-control & 46 & & & 9 & $5(55.6)$ & $31.5 \pm 8.0$ \\
\hline & & & & CD 31 & $13(41.9)$ & $33.1 \pm 7.9$ & & & \\
\hline & & & & UC 15 & $7(46.7)$ & $34.5 \pm 12.0$ & & & \\
\hline 2001 & Kanazawa S (148) & Japan & case-control & 22 & & & 20 & $12(60)$ & $60 \pm 8$ \\
\hline & & & & CD 11 & 7 (63.6) & $38.5 \pm 5.75$ & & & \\
\hline & & & & UC 11 & $6(54.5)$ & $56.5 \pm 10.75$ & & & \\
\hline 2003 & Kapsoritakis A (149) & Greece & case-control & 94 & & & 23 & & $38 \pm 9$ \\
\hline & & & & CD 44 & & & & & \\
\hline & & & & UC 50 & & & & & \\
\hline 2015 & Kleiner G (150) & Italy & case-control & $\begin{array}{l}\text { 26;CD15; } \\
\text { UC11 }\end{array}$ & $12(46.2)$ & $9 \pm 3.75$ & 37 & $22(59.5)$ & $11 \pm 4$ \\
\hline
\end{tabular}


TABLE 1 | Continued

\begin{tabular}{|c|c|c|c|c|c|c|c|c|c|}
\hline \multirow[t]{2}{*}{ Year } & \multirow[t]{2}{*}{ Author } & \multirow[t]{2}{*}{ Country } & \multirow[t]{2}{*}{ Study type } & \multicolumn{3}{|c|}{ IBD } & \multicolumn{3}{|c|}{$\mathrm{HC}$} \\
\hline & & & & Sample size & Female (\%) & Age (years) & $\begin{array}{l}\text { Sample } \\
\text { size }\end{array}$ & $\begin{array}{c}\text { Female } \\
(\%)\end{array}$ & Age (years) \\
\hline \multirow[t]{3}{*}{2004} & Magro F (151) & Portugal & case-control & 218 & & & 115 & 59 (51.3) & $32 \pm 9.75$ \\
\hline & & & & CD 145 & $84(57.9)$ & $33 \pm 14.5$ & & & \\
\hline & & & & UC 73 & $43(58.9)$ & $35 \pm 11.75$ & & & \\
\hline 2011 & Pousa ID (152) & Spain & case-control & active UC 13 & 46 & $46 \pm 12$ & 26 & & \\
\hline 2007 & Pousa ID (153) & Spain & case-control & CD 70 & $39(55.7)$ & $42 \pm 13$ & 30 & $15(50)$ & $43 \pm 14$ \\
\hline \multirow[t]{2}{*}{1997} & Schurer-Maly CC (154) & $\begin{array}{l}\text { Switzer- } \\
\text { land }\end{array}$ & case-control & CD 24 & & & 32 & & \\
\hline & & & & UC 23 & & & & & \\
\hline \multirow[t]{4}{*}{2020} & deZoeten EF (155) & America & case-control & $\begin{array}{l}\text { pediatric } \\
\text { active IBD } 17\end{array}$ & $5 / 18(27.8)$ & $12.7 \pm 12.7$ & pediatric 17 & 7/18 (38.9) & $12.7 \pm 16.5$ \\
\hline & & & & adult & & & adult 19 & 7/19 (36.8) & $56.9 \pm 14.4$ \\
\hline & & & & actuve UC 10 & $36.4 \pm 11.7$ & & & & \\
\hline & & & & inactive UC 10 & $52.6 \pm 17.7$ & & & & \\
\hline 2007 & Wiercinska-Drapalo A (156) & 6) Poland & case-control & UC 33 & $13(39.4)$ & $43 \pm 12.75$ & 20 & $5(25)$ & $38 \pm 6$ \\
\hline \multirow[t]{2}{*}{ Year } & Author & Country & Study type & & PsA & & \multicolumn{3}{|c|}{$\mathrm{HC}$} \\
\hline & & & & Sample size & Female (\%) & Age (years) & $\begin{array}{l}\text { Sample } \\
\text { size }\end{array}$ & $\begin{array}{c}\text { Female } \\
(\%)\end{array}$ & Age (years) \\
\hline 2009 & Ablin JN (14) & Israel & case-control & $\begin{array}{l}\text { skin10 } \\
\text { arthritis22 }\end{array}$ & $\begin{array}{c}4(40) \\
10(45.5)\end{array}$ & $\begin{array}{c}48.6 \pm 18.6 \\
47.18 \pm 8.15\end{array}$ & 16 & $12(75)$ & $41.69 \pm 9.71$ \\
\hline 2007 & Akman A (157) & Turkey & case-control & 46 & $30(65.2)$ & $43.2 \pm 14.4$ & 20 & 7 (35) & $34.6 \pm 14.5$ \\
\hline 2010 & Anderson KS (158) & Sweden & case-control & plaque(PV) 14 & 4 (28.6) & $47 \pm 10.75$ & 14 & & \\
\hline 2001 & Ballara S (12) & UK & cohort & arthritis 13 & 62 & $46 \pm 17.04$ & 31 & 65 & $49 \pm 12.59$ \\
\hline 2016 & Batycka-Baran A (159) & Poland & case-control & arthritis 24 & 37.5 & $48.29 \pm 9.05$ & 36 & & \\
\hline 2012 & Batycka-Baran A (160) & Poland & case-control & $\begin{array}{l}\text { plaque-type } \\
\text { psoriasis } 63\end{array}$ & 41.3 & $42.16 \pm 15.42$ & 31 & 48.4 & $41.35 \pm 15.23$ \\
\hline 2016 & Capkin AA (161) & Turkey & case-control & 48 & 16 (33.3) & $48.6 \pm 12.5$ & 48 & $20(41.7)$ & $52.3 \pm 8.4$ \\
\hline 1999 & Bhushan M (162) & UK & case-control & $\begin{array}{l}\text { chronic plaque } \\
15\end{array}$ & $6(30)$ & $45 \pm 13.75$ & 13 & $7(53.8)$ & $43 \pm 14.75$ \\
\hline 2002 & Creamer D (163) & UK & case-control & $\begin{array}{l}22 \\
\text { severe } 11 \\
\text { moderate } 11 \\
\text { arthritis } 10 \\
\text { non-arthritis } 12\end{array}$ & 7 (31.8) & $47 \pm 12$ & 17 & $7(41.2)$ & $42 \pm 10$ \\
\hline 2010 & Flisiak I (164) & Poland & case-control & $\begin{array}{l}\text { chronic plaque } \\
59 \\
\text { mild } 24 \\
\text { moderate } 20 \\
\text { severe } 15\end{array}$ & $16(27.1)$ & $49.1 \pm 2.1$ & 20 & & \\
\hline 2007 & Fink AM (165) & Austria & case-control & $\begin{array}{l}\text { arthritis } 28 \\
\text { active } 14 \\
\text { inactive } 14\end{array}$ & $\begin{array}{l}10(35.7) \\
4(28.6) \\
6(42.9)\end{array}$ & $54 \pm 13$ & 9 & $2(22.2)$ & $56 \pm 9$ \\
\hline 2012 & Kaur S (166) & Estonia & case-control & Plaque (PV) 58 & $23(39.7)$ & $41.7 \pm 12.0$ & 58 & $30(51.7)$ & $41.4 \pm 12.1$ \\
\hline 2014 & Meki AR (167) & Saudi Arabia & case-control & Plaque (PV)58 & $22(37.9)$ & $30.17 \pm 10.71$ & 22 & $11(50)$ & $29.36 \pm 8.83$ \\
\hline 2020 & Midde HS (168) & India & cohort & 54 & $16(29.6)$ & $41.28 \pm 11.83$ & 54 & $16(29.6)$ & $41.22 \pm 11.77$ \\
\hline 2002 & Nielsen HJ (169) & Denmark & cohort & Plaque (PV)16 & $9(56.25)$ & 24-70 years & 13 & & \\
\hline 2008 & Nofal A (170) & Egypt & case-control & Plaque (PV)30 & $11(37)$ & $42 \pm 12.2$ & 10 & $4(40)$ & $38.5 \pm 11.6$ \\
\hline 2015 & $\begin{array}{l}\text { Przepiera-Bedzak H } \\
(135)\end{array}$ & Poland & case-control & arthritis 69 & $39(56.5)$ & $52.0 \pm 12.0$ & 29 & $19(65.5)$ & $48.2 \pm 13.5$ \\
\hline 2016 & $\begin{array}{l}\text { Przepiera-Bedzak H } \\
(136)\end{array}$ & Poland & case-control & arthritis 76 & $43(56.6)$ & $50.8 \pm 12.7$ & 30 & $19(63.3)$ & $43.5 \pm 9.4$ \\
\hline 2013 & $\begin{array}{l}\text { Przepiera-Bedzak H } \\
(171)\end{array}$ & Poland & case-control & arthritis 80 & $43(53.8)$ & $50.1 \pm 12.0$ & 20 & $12(60)$ & $48.1 \pm 14.0$ \\
\hline 2016 & Shahidi-Dadras M (172) & Iran & case-control & $\begin{array}{l}\text { severe chronic } \\
\text { plaque } \\
\text { psoriasis } 60\end{array}$ & $27(45)$ & $38.35 \pm 14.96$ & 60 & $27(45)$ & $39.55 \pm 15.24$ \\
\hline 2016 & Shahidi-Dadras M (173) & Iran & case-control & $\begin{array}{l}\text { moderate- } \\
\text { severe chronic }\end{array}$ & $27(46.6)$ & $37.5 \pm 14.1$ & 60 & $27(45)$ & $39.6 \pm 15.2$ \\
\hline
\end{tabular}


TABLE 1 | Continued

\begin{tabular}{|c|c|c|c|c|c|c|c|c|c|}
\hline \multirow[t]{2}{*}{ Year } & \multirow[t]{2}{*}{ Author } & \multirow[t]{2}{*}{ Country } & \multirow[t]{2}{*}{ Study type } & \multicolumn{3}{|c|}{ PsA } & \multicolumn{3}{|c|}{$\mathrm{HC}$} \\
\hline & & & & Sample size & Female (\%) & Age (years) & $\begin{array}{l}\text { Sample } \\
\text { size }\end{array}$ & $\begin{array}{c}\text { Female } \\
(\%)\end{array}$ & Age (years) \\
\hline & & & & $\begin{array}{l}\text { plaque } \\
\text { psoriasis } 58\end{array}$ & & & & & \\
\hline 2009 & Takahashi H (174) & Japan & case-control & 122 & 41 (33.6) & $47.5 \pm 7.6$ & 78 & $24(30.8)$ & $38.6 \pm 12.25$ \\
\hline 2017 & Zheng YZ (175) & China & case-control & $\begin{array}{l}\text { Plaque (PV) } \\
194\end{array}$ & $74(38.1)$ & $39.5 \pm 12.70$ & 175 & 81 (46.3) & $40.2 \pm 7.58$ \\
\hline \multirow[t]{2}{*}{ Year } & Author & Country & Study type & & GD & & & $\mathrm{HC}$ & \\
\hline & & & & Sample size & Female (\%) & Age (years) & $\begin{array}{l}\text { Sample } \\
\text { size }\end{array}$ & $\begin{array}{c}\text { Female } \\
(\%)\end{array}$ & Age (years) \\
\hline 2020 & Cheng CW (10) & China & case-control & 40 & 100 & $40.9 \pm 13.5$ & 14 & 100 & $44.1 \pm 13.8$ \\
\hline 2009 & Figueroa-Vega N (176) & Spain & case-control & $\begin{array}{l}44 \\
\text { active GO } 13 \\
\text { inactive GO } 13 \\
\text { No GO } 18\end{array}$ & $\begin{array}{c}32(72.7) \\
9(69.2) \\
10(76.9) \\
13(72.2)\end{array}$ & $\begin{array}{l}45.11 \pm 15.20 \\
46.42 \pm 12.58 \\
48.77 \pm 19.31 \\
41.85 \pm 10.76\end{array}$ & 22 & $14(63.6)$ & $43.47 \pm 8.62$ \\
\hline 1998 & litaka M (177) & Japan & case-control & 49 & 39 (79.6) & $34.7 \pm 11.9$ & 37 & $26(70.3)$ & $35.7 \pm 11.2$ \\
\hline 2014 & Kajdaniuk D (178) & Poland & case-control & active G016 & $12(75)$ & $37 \pm 9$ & 22 & & \\
\hline 2016 & Rancier M (179) & Tunisia & case-control & 21 & $4(19.0)$ & $44.84 \pm 12.10$ & 55 & $29(52.7)$ & $46.36 \pm 11.03$ \\
\hline 2014 & Ye X (180) & China & case-control & $\begin{array}{l}64 \\
\text { GD } 30 \\
\text { active GO34 } \\
\text { inactive G014 }\end{array}$ & $\begin{array}{c}19(63.3) \\
23(67.6) \\
9(64.3)\end{array}$ & $\begin{array}{l}34.50 \pm 13.45 \\
31.06 \pm 15.15 \\
30.79 \pm 17.80\end{array}$ & 30 & $20(66.7)$ & $32.8 \pm 10.8$ \\
\hline
\end{tabular}

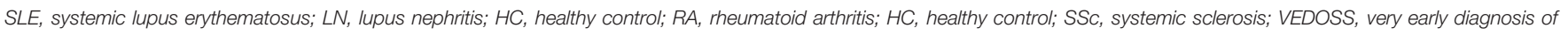
systemic sclerosis; UCTD, undifferentiated connective tissue disease; HC, healthy control; BD, Behcet's disease; HC, healthy control; KD, Kawasaki disease; HC, healthy control. AS, ankylosing spondylitis; HC, healthy control; IBD, inflammatory bowel disease; CD, Crohn's disease; UC, ulcerative colitis; HC, healthy control; PsA, psoriasis; PV, psoriasis vulgaris; HC, healthy control; GD, Graves' disease; GO, Graves' ophthalmopathy; HC, healthy control.

SLE than in inactive SLE (SMD 0.51, 95\% CI 0.33-0.70, $P<0.0001$ ) (Figure 2B-ii), whereas serum VEGF levels were significantly higher in SLE with renal involvement than that without renal involvement (SMD 1.43, 95\% CI 0.58-2.28, P = 0.0010) (Figure 2C). Due to the observed heterogeneity, the sample types were stratified (serum versus plasma); the heterogeneity in serum VEGF levels in active and inactive SLE disappeared after removing studies using plasma (before, $\mathrm{I}^{2}=$ 94.04\%, $P=0.0002$; after, $\mathrm{I}^{2}=0.00 \%, P=0.3178$ ).

The subgroup analysis indicated significantly higher serum (SMD 0.64, 95\% CI 0.37-0.91, $P<0.0001$ ) and plasma (SMD 1.56, 95\% CI 0.49-2.63, $P=0.0040$ ) VEGF levels in SLE (Figure 2D-i). Significantly higher circulating VEGF levels were present in small (n $\leq 50)$ (SMD 0.96, 95\% CI 0.56-1.35, $P<0.0001)$ and large $(\mathrm{n}>50)$ (SMD 0.39, 95\% CI 0.07-0.72, $P=0.0170$ ) studies (Figure 2D-ii).

Meta-regression analysis adjusted for age and percentage of female patients demonstrated age $(P=0.0030)$ but not sex $(P=$ 0.9700) had a significant effect.

\section{Meta-Analysis of the Association Between Circulating VEGF and RA}

Circulating VEGF levels were significantly higher in RA than in HC (SMD 1.48, 95\% CI 0.82-2.15, $P<0.0001$ ) (Figure 3A). Overall heterogeneity was apparent.

The subgroup analysis indicated significantly higher VEGF levels in serum (SMD 1.49, 95\% CI 1.09-1.88, $P<0.0001$ ) but not plasma $(P=0.0820)$ in RA (Figure 3B-i). Higher circulating VEGF levels were present in small $(\mathrm{n} \leq 50)$ (SMD 1.58, 95\% CI
$1.10-2.05, P<0.0001)$ and large $(\mathrm{n}>50)(\mathrm{SMD} 1.03,95 \% \mathrm{CI}$ $0.47-1.60, P<0.0001$ ) studies on RA (Figure 3B-ii).

Meta-regression analysis adjusted for age and female sex demonstrated neither age $(P=0.4090)$ nor sex $(P=0.7570)$ had a significant effect.

\section{Meta-Analysis of the Association Between Circulating VEGF and SSc}

Circulating VEGF levels were significantly higher in SSc than in HC (SMD 0.56, 95\% CI 0.36-0.75, $P<0.0001$ ) (Figure 4A). The comparison of serum VEGF levels between limited and diffused SSc did not reach statistical significance $(P=0.2735)$ (Figure $4 B$ ).

The subgroup analysis performed due to the obvious overall heterogeneity $\left(\mathrm{I}^{2}=98.35 \%, P<0.0001\right)$ revealed significantly higher VEGF levels in serum (SMD 0.48, 95\% CI 0.28-0.67, $P<0.0001)$ and plasma (SMD 0.86, 95\% CI 0.49-1.24, $P<0.0001)$ samples of patients with SSc (Figure 4C-i). Elevated circulating VEGF levels were observed in small ( $\mathrm{n} \leq 50)$ (SMD 0.57, 995\% CI $0.33-0.81, P<0.0001)$ and large $(\mathrm{n}>50)$ (SMD 0.52, 95\% CI $0.28-0.75, P<0.0001$ ) studies on SSc (Figure 4C-ii).

Meta-regression analysis adjusted for age and female sex demonstrated neither age $(P=0.2740)$ nor sex $(P=0.7020)$ had a significant effect.

\section{Meta-Analysis of the Association Between Circulating VEGF and BD}

Circulating VEGF levels were significantly higher in $\mathrm{BD}$ than in HC (SMD 1.65, 95\% CI 0.88-2.41, $P<0.0001$ ) (Figure 5A) as 

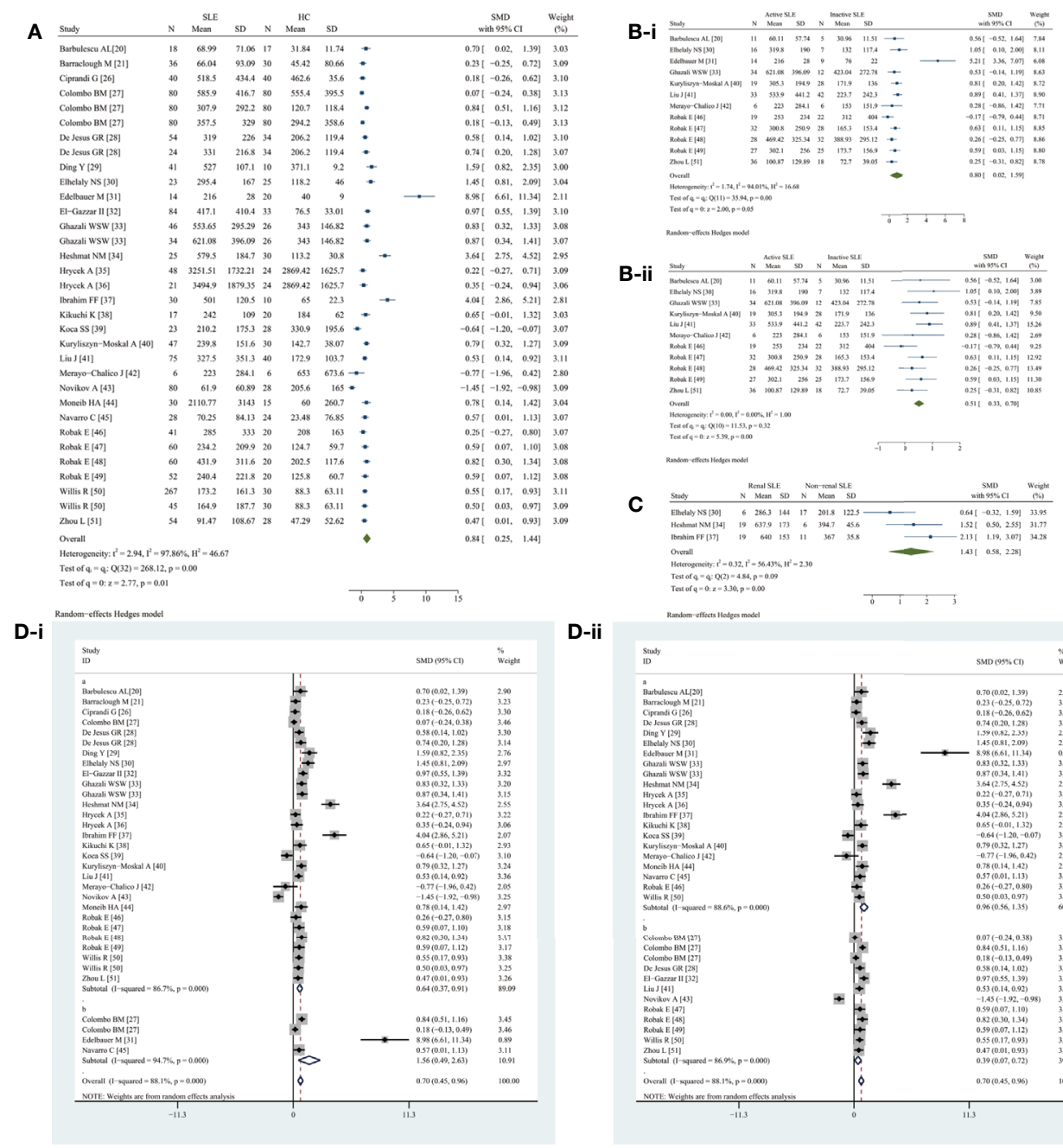

D-ii

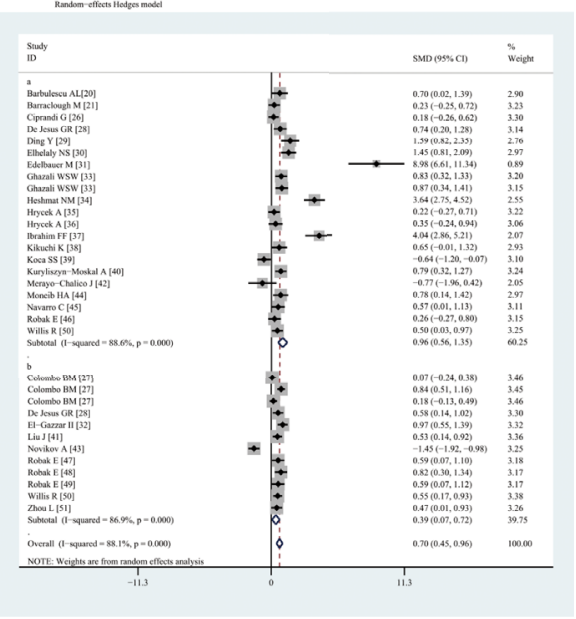

FIGURE 2 | Forest plot of SLE associated with the circulating VEGF. (A) SLE vs. HC, forest plot; (Bi) Active SLE vs. Inactive SLE; (ii) Serum VEGF in active SLE vs. inactive SLE, forest plot; (C) Renal SLE vs. Non-renal SLE, forest plot; (D) Subgroup analysis: (i) Serum vs. Plasma (a for serum and b for plasma); (ii) Sample size $n \leq 50$ vs. $n>50$ (a for $n \leq 50$ and $b$ for $n>50)$.

well as in active BD than in inactive BD (SMD 0.91, 95\% CI 0.26$1.55, P=0.0064$ ) (Figure 5B). Heterogeneity was present.

The subgroup analysis revealed significantly elevated serum VEGF levels (SMD 1.60, 95\% CI 0.85-2.34, $P<0.0001$ ) (Figure 5C-i), specifically in small $(\mathrm{n} \leq 50)(\mathrm{SMD} 1.86,95 \% \mathrm{CI}$ $1.15-2.57, P<0.0001)$ and not in large $(\mathrm{n}>50)$ studies $(P=$ 0.1200) (Figure 5C-ii).

Meta-regression analysis adjusted for age and female sex demonstrated neither age $(P=0.2700)$ nor sex $(P=0.0720)$ had a significant effect.

\section{Meta-Analysis of the Association Between Circulating VEGF and KD}

Circulating VEGF levels were elevated in KD than in HC (SMD 2.41, 95\% CI 0.10-4.72, $P=0.0406$ ) (Figure S1A) and febrile controls (SMD 1.08, 95\% CI 0.02-2.14, $P=0.0452$ ) (Figure S1B). The comparison of serum VEGF levels between acute and convalescent KD revealed no statistical significance $(P=$ 0.0831) (Figure S1C). Heterogeneity was prominent.

The subgroup analysis indicated serum VEGF levels were higher in KD than in HC (SMD 2.26, 95\% CI 0.93-3.58, P = 0.0010) (Figure S1D-i). Increased circulating VEGF levels were found in small ( $\mathrm{n} \leq 50)$ (SMD 1.36, 95\% CI 0.45-2.27, $P=0.0030)$ and large $(\mathrm{n}>50)$ studies (SMD 3.19, 95\% CI 1.01-5.38, $P=$ 0.0040) (Figure S1D-ii). Meta-regression analysis adjusted for age and female sex demonstrated female sex $(P=0.0100)$ but not age $(P=0.1280)$ had a significant effect.

\section{Meta-Analysis of the Association Between Circulating VEGF and AS}

Circulating VEGF levels were significantly elevated in AS than in HC (SMD 0.78, 95\% CI 0.23-1.33, $P=0.0052$ ) (Figure S2A). The overall heterogeneity was apparent $\left(\mathrm{I}^{2}=95.68 \%, P<0.0001\right)$. 
A

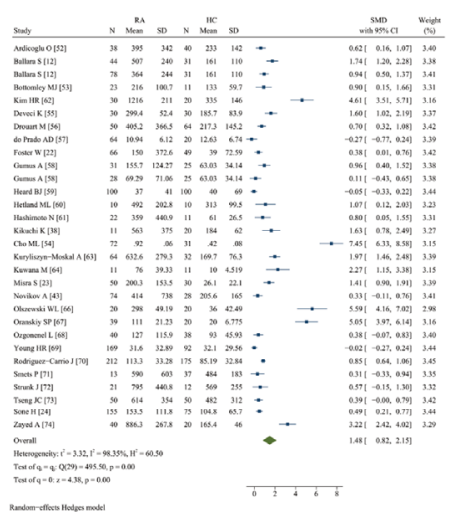

B-i

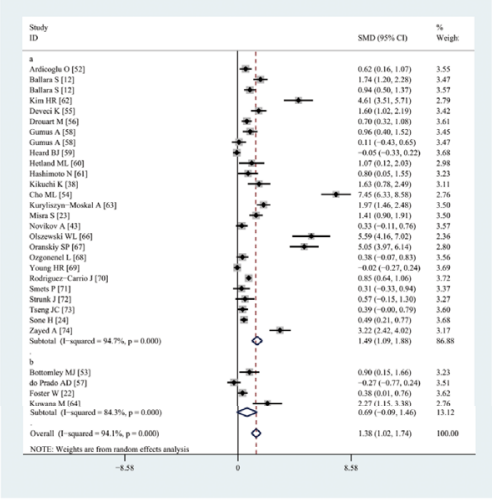

B-ii

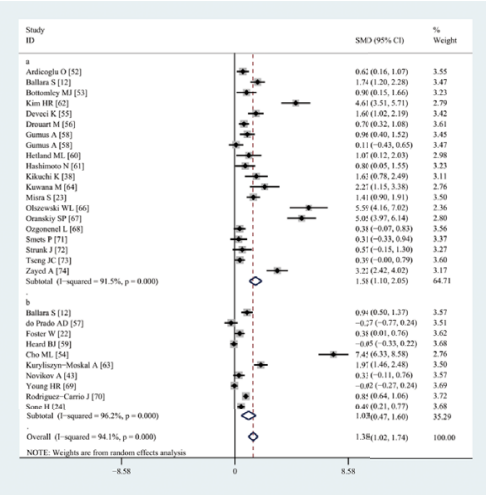

FIGURE 3 | Forest plot of RA associated with the circulating VEGF. (A) RA vs. HC, forest plot; (B) Subgroup analysis: (i) Serum vs. Plasma (a for serum and b for plasma); (ii) Sample size $n \leq 50$ vs. $n>50$ (a for $n \leq 50$ and $b$ for $n>50$ ).

A
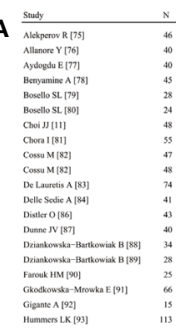

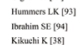

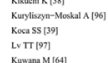

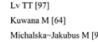

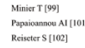

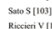

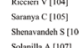

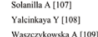

พipr)

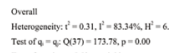

C-i

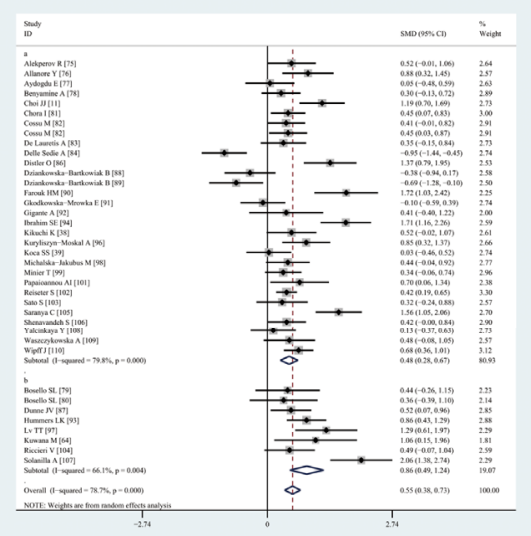

$\because \quad \begin{array}{llll}0.52[-0.02 & 1.072 & 2.67 \\ 0.851 & 0.322 & 1.37] & 2\end{array}$

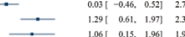

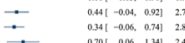

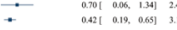

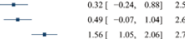

- $\quad 0.421-0.062 .0 .8412$

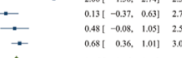

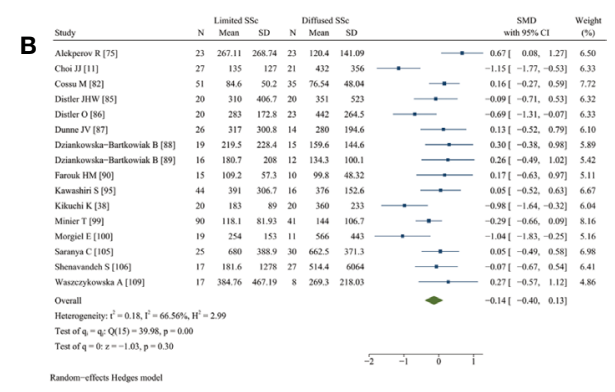

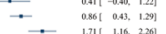

C-ii

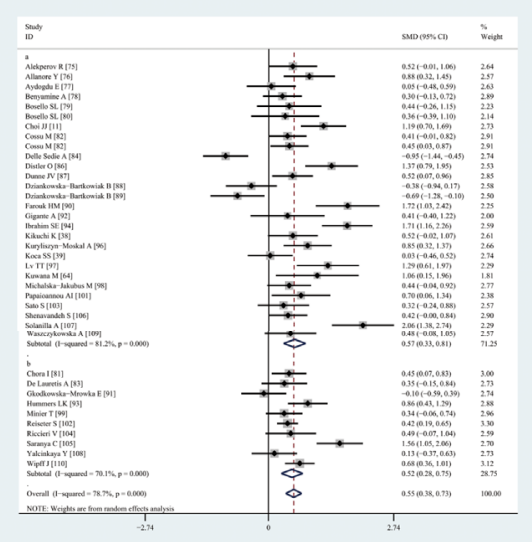

FIGURE 4 | Forest plot of SSc associated with the circulating VEGF. (A) SSc vs. HC, forest plot; (B) Limited SSc vs. Diffused SSc, forest plot; (C) Subgroup analysis: (i) Serum vs. Plasma (a for serum and b for plasma); (ii) Sample size $n \leq 50$ vs. $n>50$ (a for $n \leq 50$ and $b$ for $n>50$ ). 

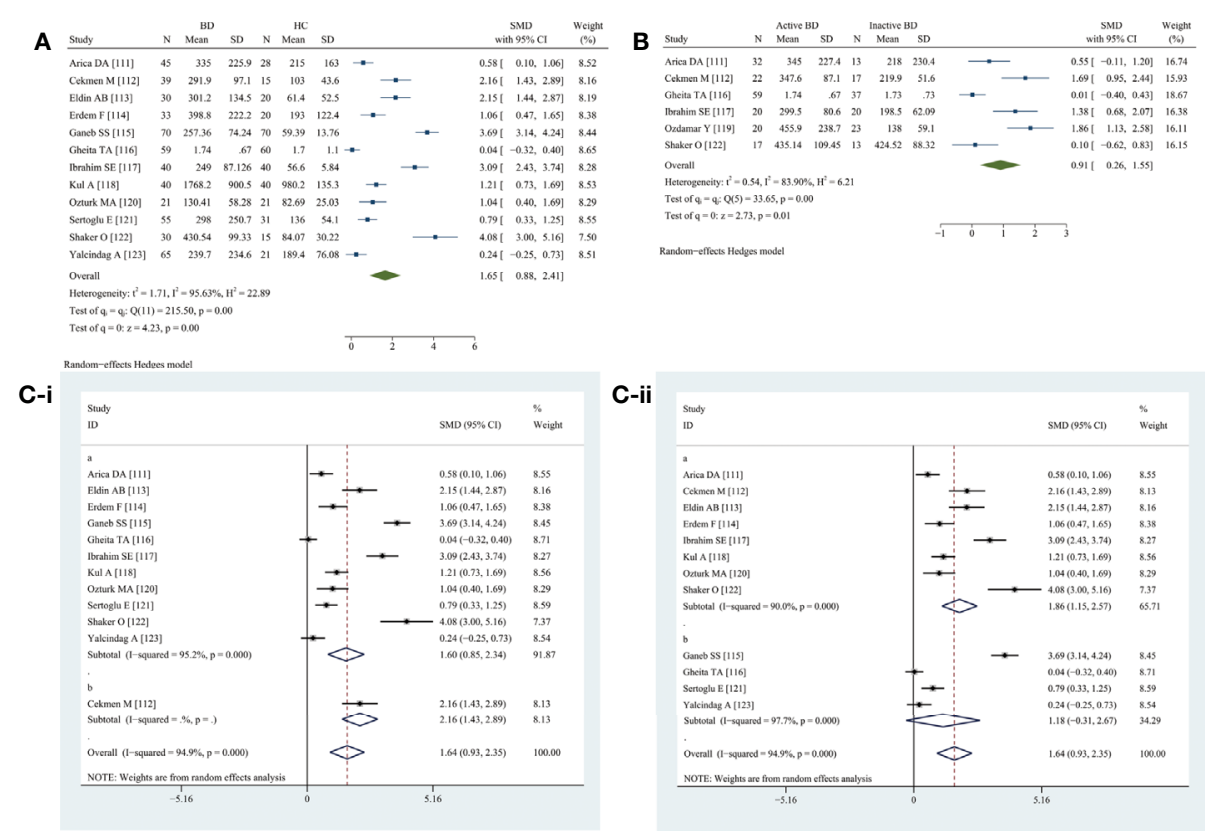

FIGURE 5 | Forest plot of BD associated with the circulating VEGF. (A) BD vs. HC, forest plot; (B) Active BD vs. Inactive BD, forest plot; (C) Subgroup analysis: (i) Serum vs. Plasma (a for serum and b for plasma); (ii) Sample size $n \leq 50$ vs. $n>50$ (a for $n \leq 50$ and $b$ for $n>50$ ).

The subgroup analysis revealed significantly higher serum VEGF levels in AS than in HC (SMD 0.60, 95\% CI 0.36-0.84, $P<0.0001)$ (Figure S2B-i). Significantly elevated circulating VEGF levels were found in small $(\mathrm{n} \leq 50)$ (SMD 1.66, 95\% CI $0.35-2.98, P=0.0130)$ and large $(\mathrm{n}>50)$ studies (SMD 0.55, 95\% CI $0.29-0.80, P<0.0001$ ) on AS (Figure S2B-ii).

Meta-regression analysis adjusted for age and female sex demonstrated neither age $(P=0.8040)$ nor sex $(P=0.8500)$ had a significant effect.

\section{Meta-Analysis of the Association Between Circulating VEGF and IBD}

Serum VEGF levels were significantly higher in IBD than in HC (SMD 0.57, 95\% CI 0.43-0.71, $P<0.0001$ ) (Figure S3A). The overall heterogeneity was extremely low $\left(\mathrm{I}^{2}=3.12 \%, P<0.0001\right)$. Meta-regression analysis adjusted for age or females demonstrated insignificant effect of age $(P=0.0760)$ and sex $(P=0.2610)$.

Serum VEGF levels were significantly higher in ulcerative colitis (UC) than in HC (SMD 0.69, 95\% CI 0.21-1.16, $P=$ 0.0048) (Figure S3B-i). Both the studies on active UC and those that did not specify disease activity reported significantly higher serum VEGF levels in UC (SMD 0.75, 95\% CI 0.17-1.34, $P=$ 0.0120 and SMD $0.56,95 \%$ CI $0.20-0.93, P=0.0030$, respectively) (Figure S3D-i). The serum VEGF levels were not significantly different between active and inactive UC $(P=$ 0.1658) (Figure S3C-i). Meta-regression analysis adjusted for age and female sex demonstrated insignificant effects of age $(P=$ $0.8330)$ and $\operatorname{sex}(P=0.2150)$.

Serum VEGF levels were significantly higher in Crohn's disease (CD) than in HC (SMD 0.72, 95\% CI 0.29-1.16,
$P=0.0011$ ) (Figure S3B-ii). Both the studies on active CD and those that did not specify disease activity reported significantly higher serum VEGF levels in CD (SMD 0.62, 95\% CI $0.10-1.15, P=0.0200$ and SMD $0.78,95 \%$ CI $0.33-1.22, P=$ 0.0010 , respectively) (Figure S3D-ii). Significantly increased serum VEGF levels were present in small ( $\mathrm{n} \leq 50)$ (SMD 0.86, 95\% CI $0.32-1.40, P=0.002)$ but not in large $(\mathrm{n}>50)$ studies $(P=$ 0.0600) (Figure S3D-iii). Moreover, serum VEGF levels were significantly higher in active CD than in inactive CD (SMD 0.53, 95\% CI 0.09-0.96, $P=0.0176$ ) (Figure S3C-ii). Meta-regression analysis adjusted for age and female sex demonstrated age $(P=$ $0.0120)$ and sex $(P=0.0010)$ had significant effects.

\section{Meta-Analysis of the Association Between Circulating VEGF and PsA}

Circulating VEGF levels were significantly higher in PsA (SMD 0.98, 95\% CI 0.62-1.34, $P<0.0001$ ) (Figure S4A), in psoriatic arthritis (SMD 0.72, 95\% CI 0.12-1.32, $P=0.0192$ ) (Figure S4B), and psoriasis with skin involvement (SMD 1.26, 95\% CI $0.65-$ $1.86, P=0.0001$ ) than in HC (Figure S4C). Heterogeneity was observed in the analyses.

The subgroup analysis indicated significantly higher serum (SMD 1.02, 95\% CI $0.50-1.55, P<0.0001$ ) and plasma (SMD $0.67,95 \%$ CI $0.37-0.97, P<0.0001$ ) VEGF levels in PsA (Figure S4D-i). Significantly higher circulating VEGF levels were found in small ( $\mathrm{n} \leq 50$ ) (SMD 0.80, 95\% CI 0.49-1.11, $P<0.0001)$ and large ( $\mathrm{n}>50)$ (SMD 1.12, 95\% CI 0.40-1.83, $P=0.0020)$ studies on PsA (Figure S4D-ii). Meta-regression analysis adjusted for age and female sex demonstrated that neither age $(P=0.0570)$ nor sex $(P=0.1890)$ had a significant effect. 


\section{Meta-Analysis of the Association Between Circulating VEGF and GD}

Circulating VEGF levels were significantly higher in GD than in HC (SMD 0.69, 95\% CI 0.20-1.19, $P=0.0056$ ), with considerable heterogeneity (Figure S5A). Circulating VEGF levels were higher in active than in inactive Graves' ophthalmopathy (GO) (SMD $0.80,95 \%$ CI $0.29-1.30, P=0.0019)$, without any heterogeneity $\left(\mathrm{I}^{2}=0.00 \%, P=0.7548\right)$ (Figure S5B).

Serum (SMD 0.77, 95\% CI 0.27-1.28, $P=0.0020$ ) but not plasma $(P=0.3880)$ VEGF levels were significantly higher in GD than in HC (Figure S5C). Meta-regression analysis adjusted for age and female sex demonstrated the significant effect of age $(P=$ $0.0070)$ but not sex $(P=0.2420)$.

\section{Correlation Analyses Between Circulation VEGF and AD Clinical Features}

We explored the potential correlation of VEGF in clinical implications and hematological indicators of ADs. For SLE (Figure S6), the summary Fisher's $\mathrm{z}$ showed a positive, moderate correlation between circulating VEGF level and disease activity (SLEDAI/SLAM, ES 0.55, 95\% CI 0.29-0.81, $P<0.0001$; summary $\mathrm{r}=0.50$ ), erythrocyte sedimentation rate (ESR; ES $0.40,95 \% \mathrm{CI}$ $0.18-0.63, P=0.0004$; summary $r=0.38)$. A negative, poor correlation was found for C3 (ES $-0.45,95 \%$ CI -0.81 to -0.08 , $P=0.0162$, summary $\mathrm{r}=-0.42$ ). There was no correlation between circulating VEGF level and platelet count $(P=0.1163)$.

In RA (Figure S7), there was a positive, weak correlation between circulating VEGF and disease activity (DAS-28; ES 0.33, 95\% CI 0.22-0.44, $P<0.0001$, summary $\mathrm{r}=0.32$ ), ESR (ES 0.35 , 95\% CI $0.18-0.51, P<0.0001$; summary $\mathrm{r}=0.34)$ as well as $\mathrm{C}-$ reactive protein (CRP; ES 0.38, 95\% CI 0.24-0.52, $P<0.0001$; summary $r=0.36)$.

In SSc (Figure S8), there was a positive, moderate relationship between circulating VEGF level and pulmonary artery pressure (ES 0.62, 95\% CI 0.37-0.87, $P<0.0001$; summary $r=0.55)$ and Medical Research Council dyspnea score (ES $0.65,95 \%$ CI $0.08-1.22, P=0.0246$; summary $\mathrm{r}=$ 0.57). There was no relationship between circulating VEGF level and modified Ronan skin score $(P=0.3100)$.

In BD (Figure S9), summary correlation coefficients indicated a significant, positive, and strong correlation with disease activity based on Behcet's disease current activity form score (ES 1.22, 95\% CI 0.03-2.41, $P=0.0446$, summary $\mathrm{r}=0.84$ ) and moderate correlation with ESR (ES 0.47, 95\% CI 0.11-0.82, $P=0.0108$, summary $\mathrm{r}=0.44)$.

In AS (Figure S10), circulating VEGF level was poorly correlated with disease activity (BASDAI/BASMI; ES 0.35, 95\% CI $0.09-0.60, P=0.0080$; summary $\mathrm{r}=0.34$ ), ESR (ES $0.26,95 \%$ CI $0.17-0.36, P<0.0001$; summary $\mathrm{r}=0.25$ ), and CRP (ES 0.24, 95\% CI 0.14-0.35, $P<0.0001$; summary $\mathrm{r}=0.24$ ).

In IBD (Figure S11), circulating VEGF level exhibited a positive, poor correlation with Crohn's disease activity index (CDAI; ES 0.34, 95\% CI 0.10-0.57, $P=0.0053$, summary $\mathrm{r}=$ 0.33 ), medium correlation with UC activity index (UDAI; ES $0.57,95 \%$ CI $0.29-0.86, P=0.0001$; summary $\mathrm{r}=0.52$ ), strong correlation with ESR (ES 0.87, 95\% CI 0.63-1.12, $P<0.0001$; summary $\mathrm{r}=0.70$ ), and weak correlation with platelet count (ES $0.32,95 \%$ CI $0.16-0.49, P=0.0001$; summary $\mathrm{r} 0.31$ ).

In PsA (Figure S12), circulating VEGF level was positively correlated with psoriasis area and severity index score (ES 1.12, 95\% CI 0.64-1.60, $P<0.0001$; summary $\mathrm{r}=0.81$ ) and had a positive, moderate correlation with disease duration (ES 0.51, 95\% CI 0.32-0.69, $P<0.0001$; summary $\mathrm{r}=0.47$ ).

\section{Sensitivity Analysis and Publication Bias}

The sensitivity analysis revealed the stability of pooled results (data not shown). For SLE, RA, SSc, KD, and AS, the contour-enhanced funnel plots revealed no publication bias (Figure S13), the metatrim practice demonstrated that all imputed studies fell into the significant region. In contrast, Egger's test suggested publication bias for SLE, RA, and KD $(P<0.0001$ for all $)$ as well as for AS $(P=$ $0.0001)$. However, there was consistency in publication bias for SSc by Egger's test $(P=0.1413)$. This remind us to be cautious with using Egger's test to determine publication bias in small number of studies $(<20)$. There was no publication bias with PsA and GD $(P$ $=0.4874$ and $P=0.5419$, respectively), in contrast to that observed with $\mathrm{BD}(P=0.0006)$. The imputed studies on IBD fell into the non-significant region, and Egger's test also represented evidence of it $(P=0.0017)$ in UC; the existence of publication bias was proven by Egger's test $(P=0.0113)$ in CD.

\section{DISCUSSION}

In the current meta-analysis, we found a close relationship between circulating VEGF level and ADs. First, our analyses revealed significantly increased circulating VEGF levels in SLE, RA, SSc, BD, KD, AS, IBD, PsA, and GD. Additionally, we showed that serum VEGF could distinguish active from inactive SLE and renal from non-renal SLE; it could also discriminate between active and inactive CD. Likewise, circulating VEGF had a strong ability to differentiate active from inactive BD and GO. Serum VEGF exhibited its dipartite boundedness in limited/ diffused cutaneous SSc, active/inactive UC, and acute/ convalescent KD. Furthermore, we demonstrated the correlation of circulating VEGF levels with metrics of disease activity and severity (SLEDAI/SLAM, DAS-28, MRC dyspnea score, modified Ronan skin score, BD current activity form score, BASDAI/BASMI, CDAI, UDAI, psoriasis area and severity index) as well as with hematological parameters (ESR, CRP, platelet count, pulmonary artery pressure). Overall, these results indicate that circulating VEGF reflects pathogenesis and should be considered as a potent hematological marker for diagnosis and disease progression in $\mathrm{ADs}$.

Structural and functional abnormities in neovasculature may lead to damage in chronic inflammatory diseases. Consecutive angiogenesis and immune-mediated vascular endothelial cell injury and dysfunction as well as persistent inflammation play important pathological roles in SLE (20), whereas expansion and invasion of synovial vessels facilitate inflammation and erosive joint destruction in RA (12). Early generalized microvascular endothelial damage leading to immune activation and defective 
angiogenesis are significant events in cumulative systemic fibrosis and microangiopathy in SSc (76). Additionally, BD is characterized by systemic vasculitis, inflammatory infiltrates, subsequent vascular lesions, and neovascularization $(113,115)$, whereas subendothelial edema and fenestrated endothelium constitute acute systemic vasculitis observed in KD (181). Structural changes in vascular endothelium due to inflammation and hypoxia stimulate angiogenesis to permeate vascular and mediate tissue repair in IBD (6). Finally, early psoriatic skin plaque formation is triggered by inappropriate expansion and vascular alterations, pronounced permeability, and endothelial cell proliferation (162). Therefore, angiogenesis and angiopathy are considered as major pathogenic events predisposing to ADs.

VEGF, an increasingly recognized proangiogenic inducer of endothelial proliferation and microvascular hyperpermeability, may reverse the tide of inducers against inhibitors and promote angiogenesis (182). Despite the unclear role of angiogenesis in AS and GD, higher-than-normal VEGF levels support its role in bone and enchondral ossification in AS (183) and increased microvessel density in GD (184). Over the past decades, numerous studies have reported increased VEGF levels in ADs, beyond its wellknown role in tumorigenesis. In the present study, our metaanalysis reveals differences in circulating VEGF levels between patients with $\mathrm{ADs}$ and $\mathrm{HC}$ subjects, providing further evidence for its utility in determining disease activity and severity in ADs.

In the present meta-analysis, there were variations in circulating VEGF levels due to differences in sample collection methods and demographic characteristics across the studies, requiring adjustment for the interpretation of the final laboratory results. Serum VEGF levels are 7-10 times higher than plasma VEGF levels in RA (60). Serum VEGF is a combination of efflux from platelets, neutrophils during coagulation, and circulating VEGF, which rarely occurs in vivo; in contrast, plasma VEGF directly reflects circulating VEGF in the absence of coagulation in vivo. In support of this difference, the present meta-analysis also revealed that the removal of plasma samples from the analysis led to the disappearance of heterogeneity in serum VEGF levels in active and inactive SLE. Plasma samples with citrate anticoagulants had the lowest VEGF levels, reflecting that that reservation of platelets VEGF releasing is effective and that different anticoagulation procedures should be considered in evaluating variations in VEGF levels across studies. Higher plasma VEGF levels in female patients compared with male patients, increasing VEGF levels with age in adults, and decreasing VEGF levels with age in children illustrate the contributory roles of sex and age to discrepancy (185). The cohort size in specific studies might also impact the mean and standard deviation. Therefore, we addressed these variables in subgroup and meta-regression analyses. The subgroup analyses explored the source of heterogeneity in serum VEGF levels for only studies on active and inactive SLE (before, $\mathrm{I}^{2}=94.04 \%, P=0.0002$; after, $\mathrm{I}^{2}=0.00 \%$, $P=0.3178)$. We also observed apparent associations of circulating VEGF levels with age and female sex in SLE and CD, with sex in $\mathrm{KD}$, and with age in GD.

There are several limitations in the present meta-analysis. First, although subgroup and meta-regression analyses were performed to explore heterogeneity, much of it remains to be explained and reported. Second, the funnel plots indicated publication bias in studies on $\mathrm{BD}$ and IBD, including UC as well as $\mathrm{CD}$, which might have led to the overestimation of pooled SMDs. Third, data could not be fully retrieved, which might have resulted in missing values in meta-regression and the omission of covariates in tests assessing heterogeneity. Availability of complete data on patient inclusion and exclusion criteria, ethnicity, AD treatment details, and exact timing and method of VEGF measurement would greatly reduce the bias in our analyses. Although the existing heterogeneity could be partially explained by age, sex, sample type, and sample size of the individual studies, an exact conclusion could not be drawn due to the lacking explanation for the remaining heterogeneity. Further studies using more comprehensive data should be performed to elucidate the association of circulating VEGF levels with ADs.

In conclusion, our meta-analysis unveiled a close association between circulating VEGF levels and ADs including disease activity and severity as well as clinical hematological manifestations. Serum VEGF is a reliable marker that can distinguish active from inactive in SLE and GO and can potentially differentiate IBD from HC. Early and regular measurement of circulating VEGF levels may be considered as a noninvasive method to monitor vascular involvement and activity in ADs. Future studies should focus on the prognostic and diagnostic utility of circulating VEGF, its role in pathogenesis, and the utility of VEGF-targeted therapeutic strategies in $\mathrm{ADs}$.

\section{DATA AVAILABILITY STATEMENT}

The original contributions presented in the study are included in the article/Supplementary Material. Further inquiries can be directed to the corresponding author.

\section{AUTHOR CONTRIBUTIONS}

YL conceived and designed the research. HZ and HL extracted data and conducted quality assessment. CL, LC, SY, HL, and HZ analyzed the data. HZ wrote the paper. All authors are accountable for all aspects of the study, and attest to the accuracy and integrity of the results. All authors contributed to the article and approved the submitted version.

\section{FUNDING}

This research was supported by grants from the National Natural Science Foundation of China Grants (81871302) and Beijing Key Clinical Specialty for Laboratory Medicine - Excellent Project (No. ZK201000).

\section{SUPPLEMENTARY MATERIAL}

The Supplementary Material for this article can be found online at: https://www.frontiersin.org/articles/10.3389/fimmu.2021. 674343/full\#supplementary-material 


\section{REFERENCES}

1. Koch AE. Angiogenesis as a Target in Rheumatoid Arthritis. Ann Rheum Dis (2003) 62(Suppl 2):ii60-7. doi: 10.1136/ard.62.suppl_2.ii60

2. Brenchley PE. Angiogenesis in Inflammatory Joint Disease: A Target for Therapeutic Intervention. Clin Exp Immunol (2000) 121:426-9. doi: 10.1046/j.1365-2249.2000.01299.x

3. Kidd BL, Moore K, Walters MT, Smith JL, Cawley MI. Immunohistological Features of Synovitis in Ankylosing Spondylitis: A Comparison With Rheumatoid Arthritis. Ann Rheum Dis (1989) 48:92-8. doi: 10.1136/ ard.48.2.92

4. Terai M, Yasukawa K, Narumoto S, Tateno S, Oana S, Kohno Y. Vascular Endothelial Growth Factor in Acute Kawasaki Disease. Am J Cardiol (1999) 83:337-9. doi: 10.1016/s0002-9149(98)00864-9

5. Ciołkiewicz M, Kuryliszyn-Moskal A, Klimiuk PA. Analysis of Correlations Between Selected Endothelial Cell Activation Markers, Disease Activity, and Nailfold Capillaroscopy Microvascular Changes in Systemic Lupus Erythematosus Patients. Clin Rheumatol (2010) 29:175-80. doi: 10.1007/ s10067-009-1308-7

6. Cibor D, Domagala-Rodacka R, Rodacki T, Jurczyszyn A, Mach T, Owczarek D. Endothelial Dysfunction in Inflammatory Bowel Diseases: Pathogenesis, Assessment and Implications. World J Gastroenterol (2016) 22:1067-77. doi: 10.3748/wjg.v22.i3.1067

7. Sakane T, Takeno M, Suzuki N, Inaba G. Behçet's Disease. N Engl J Med (1999) 341:1284-91. doi: 10.1056/nejm199910213411707

8. Sgonc R, Gruschwitz MS, Dietrich H, Recheis H, Gershwin ME, Wick G. Endothelial Cell Apoptosis is a Primary Pathogenetic Event Underlying Skin Lesions in Avian and Human Scleroderma. J Clin Invest (1996) 98:785-92. doi: 10.1172/jci118851

9. Henno A, Blacher S, Lambert CA, Deroanne C, Noël A, Lapière C, et al. Histological and Transcriptional Study of Angiogenesis and Lymphangiogenesis in Uninvolved Skin, Acute Pinpoint Lesions and Established Psoriasis Plaques: An Approach of Vascular Development Chronology in Psoriasis. J Dermatol Sci (2010) 57:162-9. doi: 10.1016/ j.jdermsci.2009.12.006

10. Cheng CW, Wu CZ, Tang KT, Fang WF, Lin JD. Simultaneous Measurement of Twenty-Nine Circulating Cytokines and Growth Factors in Female Patients With Overt Autoimmune Thyroid Diseases. Autoimmunity (2020) 53:261-9. doi: 10.1080/08916934.2020.1755965

11. Choi JJ, Min DJ, Cho ML, Min SY, Kim SJ, Lee SS, et al. Elevated Vascular Endothelial Growth Factor in Systemic Sclerosis. J Rheumatol (2003) 30 (7):1529-33.

12. Ballara S, Taylor PC, Reusch P, Marmé D, Feldmann M, Maini RN, et al. Raised Serum Vascular Endothelial Growth Factor Levels are Associated With Destructive Change in Inflammatory Arthritis. Arthritis Rheumatol (2001) 44:2055-64. doi: 10.1002/1529-0131(200109)44:9<2055::Aidart355>3.0.Co;2-2

13. Fava RA, Olsen NJ, Spencer-Green G, Yeo KT, Yeo TK, Berse B, et al. Vascular Permeability Factor/Endothelial Growth Factor (VPF/VEGF): Accumulation and Expression in Human Synovial Fluids and Rheumatoid Synovial Tissue. J Exp Med (1994) 180:341-6. doi: 10.1084/jem.180.1.341

14. Ablin JN, Goldstein Z, Aloush V, Matz H, Elkayam O, Caspi D, et al. Normal Levels and Function of Endothelial Progenitor Cells in Patients With Psoriatic Arthritis. Rheumatol Int (2009) 29:257-62. doi: 10.1007/s00296008-0676-7

15. Zhou L, Lu G, Shen L, Wang L, Wang M. Serum Levels of Three Angiogenic Factors in Systemic Lupus Erythematosus and Their Clinical Significance. BioMed Res Int (2014) 2014:627126. doi: 10.1155/2014/627126

16. Arica DA, Akşan B, Örem A, Altinkaynak BA, Yayli S, Sönmez M. High Levels of Endothelial Progenitor Cells and Circulating Endothelial Cells in Patients With Behçet's Disease and Their Relationship to Disease Activity. Bras Dermatol (2019) 94:320-6. doi: 10.1590/abd18064841.20198169

17. Aksoy EK, Çetinkaya H, Savaş B, Ensari A, Torgutalp M, Efe C. Vascular Endothelial Growth Factor, Endostatin Levels and Clinical Features Among Patients With Ulcerative Colitis and Irritable Bowel Syndrome and Among Healthy Controls: A Cross-Sectional Analytical Study. Sao Paulo Med J (2018) 136:543-50. doi: 10.1590/1516-3180.2018.0274161118
18. Ohno T, Igarashi H, Inoue K, Akazawa K, Joho K, Hara T. Serum Vascular Endothelial Growth Factor: A New Predictive Indicator for the Occurrence of Coronary Artery Lesions in Kawasaki Disease. Eur J Pediatr (2000) 159:424-9. doi: 10.1007/s004310051300

19. Lin TT, Lu J, Qi CY, Yuan L, Li XL, Xia LP, et al. Elevated Serum Level of IL27 and VEGF in Patients With Ankylosing Spondylitis and Associate With Disease Activity. Clin Exp Med (2015) 15:227-31. doi: 10.1007/s10238-0140281-X

20. Barbulescu AL, Vreju AF, Buga AM, Sandu RE, Criveanu C, Tudorascu DR, et al. Vascular Endothelial Growth Factor in Systemic Lupus Erythematosus Correlations With Disease Activity and Nailfold Capillaroscopy Changes. Romanian J Morphol Embryol (2015) 56(3):1011-6.

21. Barraclough M, McKie S, Parker B, Jackson A, Pemberton P, Elliott R, et al. Altered Cognitive Function in Systemic Lupus Erythematosus and Associations With Inflammation and Functional and Structural Brain Changes. Ann Rheumatic Dis (2019) 78:934-40. doi: 10.1136/annrheumdis2018-214677

22. Foster W, Carruthers D, Lip GYH, Blann AD. Relationships Between Endothelial, Inflammatory and Angiogenesis Markers in Rheumatoid Arthritis: Implications for Cardiovascular Pathophysiology. Thromb Res (2009) 123:659-64. doi: 10.1016/j.thromres.2008.06.014

23. Misra S, Mondal S, Chatterjee S, Guin A, Sinhamahapatra P, Ghosh A. Association of Angiogenic and Inflammatory Markers With Power Doppler Ultrasound Vascularity Grade and DAS28-CRP in Early Rheumatoid Arthritis: A Comparative Analysis. BioMed Res Int (2018) 2018:6906374. doi: 10.1155/2018/6906374

24. Sone H, Sakauchi M, Takahashi A, Suzuki H, Inoue N, Iida K, et al. Elevated Levels of Vascular Endothelial Growth Factor in the Sera of Patients With Rheumatoid Arthritis Correlation With Disease Activity. Life Sci (2001) 69:1861-9. doi: 10.1016/s0024-3205(01)01264-4

25. Wan X, Wang W, Liu J, Tong T. Estimating the Sample Mean and Standard Deviation From the Sample Size, Median, Range and/or Interquartile Range. BMC Med Res Methodol (2014) 14:135. doi: 10.1186/1471-2288-14-135

26. Ciprandi G, Murdaca G, Colombo BM, De Amici M, Marseglia GL. Serum Vascular Endothelial Growth Factor in Allergic Rhinitis and Systemic Lupus Erythematosus. Hum Immunol (2008) 69:510-2. doi: 10.1016/ j.humimm.2008.05.010

27. Colombo BM, Cacciapaglia F, Puntoni M, Murdaca G, Rossi E, Rodriguez G, et al. Traditional and non Traditional Risk Factors in Accelerated Atherosclerosis in Systemic Lupus Erythematosus: Role of Vascular Endothelial Growth Factor (VEGATS Study). Autoimmun Rev (2009) 8:309-15. doi: 10.1016/j.autrev.2008.10.002

28. De Jesus GR, Oliveira CS, Dos Santos FC, De Jesus NR, Porto LC, Levy RA, et al. Angiogenic and Antiangiogenic Factors in Patients With Systemic Lupus Erythematosus. Arthritis Rheumatol (2014) 66:S1157. doi: 10.1002/ art.38914

29. Ding Y, Liao W, Yi ZW, Xiang W, He XJ. Association of miRNA-145 Expression in Vascular Smooth Muscle Cells With Vascular Damages in Patients With Lupus Nephritis. Int J Clin Exp Pathol (2015) 8(10):12646-56.

30. Elhelaly NS, Elhawary IM, Alaziz IAA, Alsalam MIA, Elfishawy HM, Sherif MM. The Clinical Utility of Vascular Endothelial Growth Factor as Predictive Marker for Systemic Lupus Erythematosus Activity in Children and Adolescents. J Biol Sci (2009) 9:549-54. doi: 10.3923/jbs.2009.549.554

31. Edelbauer M, Kshirsagar S, Riedl M, Billing H, Tonshoff B, Haffner D, et al. Soluble VEGF Receptor 1 Promotes Endothelial Injury in Children and Adolescents With Lupus Nephritis. Pediatr Nephrol (2012) 27:793-800. doi: 10.1007/s00467-011-2062-z

32. El-Gazzar II, Ibrahim SE, El-Sawy WS, Fathi HM, Eissa AH. Assessment of Vascular Endothelial Growth Factor in Systemic Lupus Erythematosus Patients With Anti-Phospholipid Syndrome. Egyptian Rheumatol (2019) 41:41-5. doi: 10.1016/j.ejr.2018.03.005

33. Ghazali WSW, Iberahim R, Ashari NSM. Serum Vascular Endothelial Growth Factor (VEGF) as a Biomarker for Disease Activity in Lupus Nephritis. Malays J Med Sci (2017) 24:62-72. doi: 10.21315/mjms2017.24.5.7

34. Heshmat NM, El-Kerdany TH. Serum Levels of Vascular Endothelial Growth Factor in Children and Adolescents With Systemic Lupus Erythematosus. Pediatr Allergy Immunol (2007) 18:346-53. doi: 10.1111/ j.1399-3038.2006.00510.x 
35. Hrycek A, Janowska J, Cieslik P. Selected Angiogenic Cytokines in Systemic Lupus Erythematosus Patients. Autoimmunity (2009) 42:459-66. doi: 10.1080/08916930902960339

36. Hrycek A, Pierzchała W, Osławska-Dzierżęga A, Cieślik P. Selected Growth Factors and Diffusing Capacity of the Lung for Carbon Monoxide in Patients With Systemic Lupus Erythematosus. Rheumatol Int (2009) 30:175-9. doi: 10.1007/s00296-009-0930-7

37. Ibrahim FF, Draz HM, Al Sherbeni HH. Serum Levels of Vascular Endothelial Growth Factor and Hemoglobin Dielectric Properties in Patients With Systemic Lupus Erythematosus. J Med Sci (2008) 8:469-76. doi: $10.3923 /$ jms.2008.469.476

38. Kikuchi K, Kubo M, Kadono T, Yazawa N, Ihn H, Tamaki K. Serum Concentrations of Vascular Endothelial Growth Factor in Collagen Diseases. Br J Dermatol (1998) 139:1049-51. doi: 10.1046/j.1365-2133.1998.02563.x

39. Koca SS, Akbas F, Ozgen M, Yolbas S, Ilhan N, Gundogdu B, et al. Serum Galectin-3 Level in Systemic Sclerosis. Clin Rheumatol (2014) 33:215-20. doi: 10.1007/s10067-013-2346-8

40. Kuryliszyn-Moskal A, Klimiuk PA, Sierakowski S, Ciolkiewicz M. Vascular Endothelial Growth Factor in Systemic Lupus Erythematosus: Relationship to Disease Activity, Systemic Organ Manifestation, and Nailfold Capillaroscopic Abnormalities. Archivum Immunol Et Ther Experiment (2007) 55:179-85. doi: 10.1007/s00005-007-0017-7

41. Liu J, Wang X, Yang X, Yan Q, Wang S, Han W. Investigating the Role of Angiogenesis in Systemic Lupus Erythematosus. Lupus (2015) 24:621-7. doi: $10.1177 / 0961203314556293$

42. Merayo-Chalico J, Barrera-Vargas A, Juarez-Vega G, Alcocer-Varela J, Arauz A, Gomez-Martin D. Differential Serum Cytokine Profile in Patients With Systemic Lupus Erythematosus and Posterior Reversible Encephalopathy Syndrome. Clin Exp Immunol (2018) 192:165-70. doi: 10.1111/cei.13095

43. Novikov A, Aleksandrova E, Verizhnikova Z, Panafidina T, Popkova T, Karateev D, et al. Cytokine Profiles in Systemic Lupus Erythematosus and Rheumatoid Arthritis. Ann Rheumatic Dis (2016) 75:907. doi: 10.1136/ annrheumdis-2016-eular.3130

44. Moneib HA, Salem SAM, Aly DG, Khedr HTM, Wafaey HA, Hassan HE. Assessment of Serum Vascular Endothelial Growth Factor and Nail Fold Capillaroscopy Changes in Systemic Lupus Erythematosus With and Without Cutaneous Manifestations. J Dermatol (2012) 39:52-7. doi: 10.1111/j.1346-8138.2011.01322.x

45. Navarro C, Candia-Zúñiga L, Silveira LH, Ruiz V, Gaxiola M, Avila MC, et al. Vascular Endothelial Growth Factor Plasma Levels in Patients With Systemic Lupus Erythematosus and Primary Antiphospholipid Syndrome. Lupus (2002) 11:21-4. doi: 10.1191/0961203302lu131oa

46. Robak E, Wierzbowska A, Chmiela M, Kulczycka L, Sysa-Jedrejowska A, Robak T. Circulating Total and Active Metalloproteinase-9 and Tissue Inhibitor of Metalloproteinases-1 in Patients With Systemic Lupus Erythomatosus. Mediators Inflamm (2006) 2006:17898. doi: 10.1155/mi/ 2006/17898

47. Robak E, Sysa-Jedrzejewska A, Robak T. Vascular Endothelial Growth Factor and its Soluble Receptors VEGFR-1 and VEGFR-2 in the Serum of Patients With Systemic Lupus Erythematosus. Mediators Inflamm (2003) 12:293-8. doi: 10.1080/09629350310001619726

48. Robak E, Kulczycka-Siennicka L, Gerlicz Z, Kierstan M, Korycka-Wolowiec A, Sysa-Jedrzejowska A. Correlations Between Concentrations of Interleukin (IL)-17A, IL-17B and IL-17F, and Endothelial Cells and Proangiogenic Cytokines in Systemic Lupus Erythematosus Patients. Eur Cytokine Netw (2013) 24:60-8. doi: 10.1684/ecn.2013.0330

49. Robak E, Woźniacka A, Sysa-Jedrzejowska A, Stepień H, Robak T. Circulating Angiogenesis Inhibitor Endostatin and Positive Endothelial Growth Regulators in Patients With Systemic Lupus Erythematosus. Lupus (2002) 11:348-55. doi: 10.1191/0961203302lu199oa

50. Willis R, Smikle M, DeCeulaer K, Romay-Penabad Z, Papalardo E, Jajoria P, et al. Clinical Associations of Proinflammatory Cytokines, Oxidative Biomarkers and Vitamin D Levels in Systemic Lupus Erythematosus. Lupus (2017) 26:1517-27. doi: 10.1177/0961203317706557

51. Zhou L, Lu GY, Shen L, Wang LF, Wang MJ. Serum Levels of Three Angiogenic Factors in Systemic Lupus Erythematosus and Their Clinical Significance. BioMed Res Int (2014) 2014:627126. doi: 10.1155/2014/627126
52. Ardicoglu O, Boz K, Kamanli A, Kavuncu V, Canatan H, Onal S, et al. Levels of Vascular Endothelial Growth Factor in Patients With Rheumatoid Arthritis. Pain Clin (2004) 16:187-91. doi: 10.1163/156856904774134406

53. Bottomley MJ, Webb NJ, Watson CJ, Holt L, Bukhari M, Denton J, et al. Placenta Growth Factor (PIGF) Induces Vascular Endothelial Growth Factor (VEGF) Secretion From Mononuclear Cells and is Co-Expressed With VEGF in Synovial Fluid. Clin Exp Immunol (2000) 119:182-8. doi: 10.1046/j.1365-2249.2000.01097.x

54. Cho ML, Jung YO, Moon YM, Min SY, Yoon CH, Lee SH, et al. Interleukin18 Induces the Production of Vascular Endothelial Growth Factor (VEGF) in Rheumatoid Arthritis Synovial Fibroblasts. Via AP-1Dependent Pathways Immunol Lett (2006) 103:159-66. doi: 10.1016/ j.imlet.2005.10.020

55. Deveci K, Butun I, Deveci H. Evaluation of Nitric Oxide and Angiogenic Factors in Rheumatoid Arthritis and Ankylosing Spondylitis Patients. FEBS J (2016) 283:244-5. doi: 10.1111/febs.13808

56. Drouart M, Saas P, Billot M, Cedoz JP, Tiberghien P, Wendling D, et al. High Serum Vascular Endothelial Growth Factor Correlates With Disease Activity of Spondylarthropathies. Clin Exp Immunol (2003) 132:158-62. doi: 10.1046/j.1365-2249.2003.02101.x

57. do Prado AD, Bisi MC, Piovesan DM, Bredemeier M, Batista TS, Petersen L, et al. Ultrasound Power Doppler Synovitis is Associated With Plasma IL-6 in Established Rheumatoid Arthritis. Cytokine (2016) 83:27-32. doi: 10.1016/ j.cyto.2016.03.014

58. Gumus A, Coskun C, Emre HO, Temel M, Inal BB, Seval H, et al. Evaluation of Vascular Endothelial Growth Factor Levels in Rheumatoid Arthritis Patients, With and Without Joint Swelling; a Comparison With Erythrocyte Sedimentation Rate, C-reactive Protein, Rheumatoid Factor and Anti-Cyclic Citruillnated Protein. Turkish J Biochem-Turk Biyokim Dergisi (2018) 43:76-82. doi: 10.1515/tjb-2016-0308

59. Heard BJ, Rosvold JM, Fritzler MJ, El-Gabalawy H, Wiley JP, Krawetz RJ. A Computational Method to Differentiate Normal Individuals, Osteoarthritis and Rheumatoid Arthritis Patients Using Serum Biomarkers. $J$ R Soc Interface (2014) 11:20140428. doi: 10.1098/rsif.2014.0428

60. Hetland ML, Christensen IJ, Lottenburger T, Johansen JS, Svendsen MN, Horslev-Petersen K, et al. Circulating VEGF as a Biological Marker in Patients With Rheumatoid Arthritis? Preanalytical and Biological Variability in Healthy Persons and in Patients. Dis Markers (2008) 24:1-10. doi: $10.1155 / 2008 / 707864$

61. Hashimoto N, Iwasaki T, Kitano M, Ogata A, Hamano T. Levels of Vascular Endothelial Growth Factor and Hepatocyte Growth Factor in Sera of Patients With Rheumatic Diseases. Mod Rheumatol (2003) 13:129-34. doi: 10.3109/s10165-002-0211-8

62. Kim HR, Park MK, Cho ML, Yoon CH, Lee SH, Park SH, et al. Macrophage Migration Inhibitory Factor Upregulates Angiogenic Factors and Correlates With Clinical Measures in Rheumatoid Arthritis. J Rheumatol (2007) 34 (5):927-36.

63. Kuryliszyn-Moskal A, Klimiuk PA, Sierakowski S, Ciolkiewicz M. A Study on Vascular Endothelial Growth Factor and Endothelin-1 in Patients With Extra-Articular Involvement of Rheumatoid Arthritis. Clin Rheumatol (2006) 25:314-9. doi: 10.1007/s10067-005-0007-2

64. Kuwana M, Okazaki Y, Yasuoka H, Kawakami Y, Ikeda Y. Defective Vasculogenesis in Systemic Sclerosis. Lancet (2004) 364:603-10. doi: 10.1016/s0140-6736(04)16853-0

65. Milman N, Karsh J, Booth RA. Correlation of a Multi-Cytokine Panel With Clinical Disease Activity in Patients With Rheumatoid Arthritis. Clin Biochem (2010) 43:1309-14. doi: 10.1016/j.clinbiochem.2010.07.012

66. Olszewski WL, Pazdur J, Kubasiewicz E, Zaleska M, Cooke CJ, Miller NE. Lymph Draining From Foot Joints in Rheumatoid Arthritis Provides Insight Into Local Cytokine and Chemokine Production and Transport to Lymph Nodes. Arthritis Rheumatol (2001) 44:541-9. doi: 10.1002/1529-0131 (200103)44:3<541::Aid-anr102>3.0.Co;2-6

67. Oranskiy SP, Yeliseyeva LN, Tsanaeva AV, Zaytseva NV. Body Composition and Serum Levels of Adiponectin, Vascular Endothelial Growth Factor, and Interleukin-6 in Patients With Rheumatoid Arthritis. Croatian Med J (2012) 53:350-6. doi: $10.3325 / \mathrm{cmj} .2012 .53 .350$

68. Ozgonenel L, Cetin E, Tutun S, Tonbaklar P, Aral H, Guvenen G. The Relation of Serum Vascular Endothelial Growth Factor Level With Disease 
Duration and Activity in Patients With Rheumatoid Arthritis. Clin Rheumatol (2010) 29:473-7. doi: 10.1007/s10067-009-1343-4

69. Young HR, Chung CP, Oeser A, Solus J, Asanuma Y, Sokka T, et al. Inflammatory Mediators and Premature Coronary Atherosclerosis in Rheumatoid Arthritis. Arthritis Care Res (2009) 61:1580-5. doi: 10.1002/ art.25009

70. Rodriguez-Carrio J, Alperi-Lopez M, Lopez-Mejias R, Lopez P, BallinaGarcia FJ, Abal F, et al. Antibodies to Paraoxonase 1 are Associated With Oxidant Status and Endothelial Activation in Rheumatoid Arthritis. Clin Sci (2016) 130:1889-99. doi: 10.1042/cs20160374

71. Smets P, Devauchelle-Pensec V, Rouzaire PO, Pereira B, Andre M, Soubrier M. Vascular Endothelial Growth Factor Levels and Rheumatic Diseases of the Elderly. Arthritis Res Ther (2016) 18:283. doi: 10.1186/s13075-016-1184-x

72. Strunk J, Heinemann E, Neeck G, Schmidt KL, Lange U. A New Approach to Studying Angiogenesis in Rheumatoid Arthritis by Means of Power Doppler Ultrasonography and Measurement of Serum Vascular Endothelial Growth Factor. Rheumatol (Oxford) (2004) 43:1480-3. doi: 10.1093/rheumatology/ keh380

73. Tseng JC, Keng HM, Yen LJ, Wang LF, Hu RJ, Lu LY. Analysis of Serum Vascular Endothelial Growth Factor and Angiogenesis Inhibitor Endostatin Concentrations in Patients With Different Arthritis. Int J Rheumatic Dis (2010) 13:79. doi: 10.1111/j.1756-185X.2010.01497.x

74. Zayed A, Amer H, Nasar H, Ibrahiem A, Mottawie H. Some Biochemical Changes in Serum and Synovial Fluid of Patients With Rheumatoid Arthritis. J Med Sci (2007) 7:526-35. doi: 10.3923/jms.2007.526.535

75. Alekperov R, Alexandrova E, Novikov A, Ananyeva L. Clinical Associations of Vascular Endothelial Growth Factor And its Receptor 2 Type in Systemic Sclerosis. Ann Rheumatic Dis (2018) 77:1531-. doi: 10.1136/annrheumdis2018-eular.4064

76. Allanore Y, Borderie D, Lemaréchal H, Ekindjian OG, Kahan A. Nifedipine Decreases sVCAM-1 Concentrations and Oxidative Stress in Systemic Sclerosis But Does Not Affect the Concentrations of Vascular Endothelial Growth Factor or its Soluble Receptor 1. Arthritis Res Ther (2004) 6:R30914. doi: $10.1186 /$ ar1 183

77. Aydogdu E, Pamuk ON, Donmez S, Pamuk GE. Decreased interleukin-20 Level in Patients With Systemic Sclerosis: Are They Related With Angiogenesis? Clin Rheumatol (2013) 32:1599-603. doi: 10.1007/s10067-013-2317-0

78. Benyamine A, Magalon J, Cointe S, Lacroix R, Arnaud L, Bardin N, et al. Increased Serum Levels of Fractalkine and Mobilisation of CD34+CD45Endothelial Progenitor Cells in Systemic Sclerosis. Arthritis Res Ther (2017) 19:60. doi: 10.1186/s13075-017-1271-7

79. Bosello SL, Canestrari G, Capacci A, Di Giorgio A, De Luca G, Bocci M, et al. Fmd, VEGF, and IL-6 in Vedoss Patients: The Precocity of Endothelial Dysfunction. Ann Rheumatic Dis (2014) 73. doi: 10.1136/annrheumdis2014-eular.4446

80. Bosello SL, Di Giorgio A, Foti F, De Luca G, Bocci M, Parisi F, et al. Decrease of Brachial Artery Endothelial-Dependent Flow-Mediated Dilation Characterizes Very Early Systemic Sclerosis (VEDOSS) Patients. Clin Exp Rheumatol (2014) 32:S23.

81. Chora I, Romano E, Manetti M, Mazzotta C, Costa R, Machado V, et al. Evidence for a Derangement of the Microvascular System in Patients With a Very Early Diagnosis of Systemic Sclerosis. J Rheumatol (2017) 44:1190-7. doi: 10.3899/jrheum. 160791

82. Cossu M, Andracco R, Santaniello A, Marchini M, Severino A, Caronni M, et al. Serum Levels of Vascular Dysfunction Markers Reflect Disease Severity and Stage in Systemic Sclerosis Patients. Rheumatology (2016) 55:1112-6. doi: 10.1093/rheumatology/kew017

83. De Lauretis A, Sestini P, Pantelidis P, Hoyles R, Hansell DM, Goh NSL, et al. Serum Interleukin 6 Is Predictive of Early Functional Decline and Mortality in Interstitial Lung Disease Associated With Systemic Sclerosis. J Rheumatol (2013) 40:435-46. doi: 10.3899/jrheum.120725

84. Delle Sedie A, Riente L, Maggiorini L, Pratesi F, Tavoni A, Migliorini P, et al. Potential Biomarkers in Patients With Systemic Sclerosis. Int J Rheum Dis (2018) 21:261-5. doi: 10.1111/1756-185x.13196

85. Distler JHW, Strapatsas T, Huscher D, Dees C, Akhmetshina A, Kiener HP, et al. Dysbalance of Angiogenic and Angiostatic Mediators in Patients With Mixed Connective Tissue Disease. Ann Rheumatic Dis (2011) 70:1197-202. doi: 10.1136/ard.2010.140657
86. Distler O, Del Rosso A, Giacomelli R, Cipriani P, Conforti ML, Guiducci S, et al. Angiogenic and Angiostatic Factors in Systemic Sclerosis: Increased Levels of Vascular Endothelial Growth Factor are a Feature of the Earliest Disease Stages and are Associated With the Absence of Fingertip Ulcers. Arthritis Res (2002) 4:R11. doi: 10.1186/ar596

87. Dunne JV, Keen KJ, Van Eeden SF. Circulating Angiopoietin and Tie-2 Levels in Systemic Sclerosis. Rheumatol Int (2013) 33:475-84. doi: 10.1007/ s00296-012-2378-4

88. Dziankowska-Bartkowiak B, Waszczykowska E, Zalewska A, SysaJedrzejowska A. Correlation of Endostatin and Tissue Inhibitor of Metalloproteinases 2 (TIMP2) Serum Levels With Cardiovascular Involvement in Systemic Sclerosis Patients. Mediators Inflamm (2005) 2005:144-9. doi: 10.1155/mi.2005.144

89. Dziankowska-Bartkowiak B, Waszczykowska E, Dziankowska-Zaboroszczyk E, de Graft-Johnson JE, Zalewska A, Łuczyńska M, et al. Decreased Ratio of Circulatory Vascular Endothelial Growth Factor to Endostatin in Patients With Systemic Sclerosis-Association With Pulmonary Involvement. Clin Exp Rheumatol (2006) 24(5):508-13.

90. Farouk HM, Hamza SH, El Bakry SA, Youssef SS, Aly IM, Moustafa AA, et al. Dysregulation of Angiogenic Homeostasis in Systemic Sclerosis. Int $J$ Rheumatic Dis (2013) 16:448-54. doi: 10.1111/1756-185x.12130

91. Gkodkowska-Mrowka E, Gorska E, Ciurzynski M, Stelmaszczyk-Emmel A, Bienias P, Irzyk K, et al. Pro- and Antiangiogenic Markers in Patients With Pulmonary Complications of Systemic Scleroderma. Respir Physiol

Neurobiol (2015) 209:69-75. doi: 10.1016/j.resp.2014.10.018

92. Gigante A, Navarini L, Margiotta D, Barbano B, Afeltra A, Rosato E. Female Sexual Dysfunction in Systemic Sclerosis: The Role of Endothelial Growth Factor and Endostatin. J Scleroderma Related Disord (2019) 4:71-6. doi: $10.1177 / 2397198318776593$

93. Hummers LK, Hall A, Wigley FM, Simons M. Abnormalities in the Regulators of Angiogenesis in Patients With Scleroderma. J Rheumatol (2009) 36:576-82. doi: 10.3899/jrheum.080516

94. Ibrahim SE, Morad CS, Farouk N, Louis A. Platelet Indices as Markers of Inflammation in Systemic Sclerosis Patients: Relation to Vascular Endothelial Growth Factor and Flow Mediated Dilatation. Egyptian Rheumatol (2018) 40:239-42. doi: 10.1016/j.ejr.2017.12.001

95. Kawashiri S, Nishino A, Igawa T, Takatani A, Shimizu T, Umeda M, et al. Prediction of Organ Involvement in Systemic Sclerosis by Serum Biomarkers and Peripheral Endothelial Function. Clin Exp Rheumatol (2018) 36(4): S102-S8.

96. Kuryliszyn-Moskal A, Klimiuk PA, Sierakowski S. Soluble Adhesion Molecules (sVCAM-1, sE-selectin), Vascular Endothelial Growth Factor (VEGF) and Endothelin-1 in Patients With Systemic Sclerosis: Relationship to Organ Systemic Involvement. Clin Rheumatol (2005) 24:111-6. doi: 10.1007/s10067-004-0987-3

97. Lv TT, Yang FF, Zhang K, Lv MH, Zhang Y, Zhu P. The Risk of Circulating Angiogenic T Cells and Subsets in Patients With Systemic Sclerosis. Int Immunopharmacol (2020) 81:106282. doi: 10.1016/j.intimp.2020.106282

98. Michalska-Jakubus M, Cutolo M, Smith V, Krasowska D. Imbalanced Serum Levels of Ang1, Ang2 and VEGF in Systemic Sclerosis: Integrated Effects on Microvascular Reactivity. Microvascular Res (2019) 125:103881. doi: 10.1016/j.mvr.2019.103881

99. Minier T, Nagy Z, Balint Z, Farkas H, Radics J, Kumanovics G, et al. Construct Validity Evaluation of the European Scleroderma Study Group Activity Index, and Investigation of Possible New Disease Activity Markers in Systemic Sclerosis. Rheumatology (2010) 49:1133-45. doi: 10.1093/ rheumatology/keq022

100. Morgiel E, Wiland G, Piesiak P, Bogut B, Wiland P. VEGF Serum Levels as an Indicator of a Visceral Involvement in SSc. Rheumatology (2012) 51:ii75. doi: 10.1093/rheumatology/ker475

101. Papaioannou AI, Zakynthinos E, Kostikas K, Kiropoulos T, Koutsokera A, Ziogas A, et al. Serum VEGF Levels are Related to the Presence of Pulmonary Arterial Hypertension in Systemic Sclerosis. BMC Pulm Med (2009) 9:18. doi: 10.1186/1471-2466-9-18

102. Reiseter S, Molberg O, Gunnarsson R, Lund MB, Aalokken TM, Aukrust P, et al. Associations Between Circulating Endostatin Levels and Vascular 
Organ Damage in Systemic Sclerosis and Mixed Connective Tissue Disease: An Observational Study. Arthritis Res Ther (2015) 17(1):231. doi: 10.1186/ s13075-015-0756-5

103. Sato S, Hasegawa M, Takehara K. Serum Levels of Interleukin-6 and interleukin-10 Correlate With Total Skin Thickness Score in Patients With Systemic Sclerosis. J Dermatol Sci (2001) 27:140-6. doi: 10.1016/s0923-1811 (01)00128-1

104. Riccieri V, Stefanantoni K, Vasile M, Macri V, Sciarra I, Iannace N, et al. Abnormal Plasma Levels of Different Angiogenic Molecules are Associated With Different Clinical Manifestations in Patients With Systemic Sclerosis. Clin Exp Rheumatol (2011) 29(2):S46-52.

105. Saranya C, Ramesh R, Bhuvanesh M, Balaji C, Balameena S, Rajeswari S. Serum Vascular Endothelial Growth Factor Levels as a Marker of Skin Thickening, Digital Ischemia, and Interstitial Lung Disease in Systemic Sclerosis. Indian J Rheumatol (2018) 13:182-5. doi: 10.4103/injr.injr_132_17

106. Shenavandeh S, Tarakemeh T, Sarvestani EK, Nazarinia MA. Serum Vascular Endothelial Growth Factor (VEGF), Soluble VEGF Receptor-1 (sVEGFR-1) and sVEGFR-2 in Systemic Sclerosis Patients: Relation to Clinical Manifestations and Capillaroscopy Findings. Egyptian Rheumatol (2017) 39:19-24. doi: 10.1016/j.ejr.2016.03.004

107. Solanilla A, Villeneuve J, Auguste P, Hugues M, Alioum A, Lepreux S, et al. The Transport of High Amounts of Vascular Endothelial Growth Factor by Blood Platelets Underlines Their Potential Contribution in Systemic Sclerosis Angiogenesis. Rheumatology (2009) 48:1036-44. doi: 10.1093/ rheumatology/kep154

108. Yalcinkaya Y, Adin-Cinar S, Artim-Esen B, Kamali S, Pehlivan O, Ocal L, et al. Capillaroscopic Findings and Vascular Biomarkers in Systemic Sclerosis: Association of Low CD40L Levels With Late Scleroderma Pattern. Microvascular Res (2016) 108:17-21. doi: 10.1016/j.mvr.2016.07.002

109. Waszczykowska A, Gos R, Waszczykowska E, Dziankowska-Bartkowiak B, Podgorski M, Jurowski P. The Role of Angiogenesis Factors in the Formation of Vascular Changes in Scleroderma by Assessment of the Concentrations of VEGF and sVEGFR2 in Blood Serum and Tear Fluid. Mediators Inflammation (2020) 2020. doi: 10.1155/2020/7649480

110. Wipff J, Avouac J, Borderie D, Zerkak D, Lemarechal H, Kahan A, et al. Disturbed Angiogenesis in Systemic Sclerosis: High Levels of Soluble Endoglin. Rheumatology (2008) 47:972-5. doi: 10.1093/rheumatology/ ken100

111. Arica DA, Aksan B, Orem A, Altinkaynak BA, Yayli S, Sonmez M. High Levels of Endothelial Progenitor Cells and Circulating Endothelial Cells in Patients With Behcet's Disease and Their Relationship to Disease Activity. Anais Brasileiros Dermatol (2019) 94:320-6. doi: 10.1590/abd18064841.20198169

112. Cekmen M, Evereklioglu C, Er H, Inalöz HS, Doganay S, Türköz Y, et al. Vascular Endothelial Growth Factor Levels are Increased and Associated With Disease Activity in Patients With Behçet's Syndrome. Int J Dermatol (2003) 42:870-5. doi: 10.1046/j.1365-4362.2003.01688.x

113. Eldin AB, Ibrahim A. Assessment of the Relationship Between Vascular Endothelial Growth Factor and Cardiovascular Involvement in Egyptian Patients With Behçet's Disease. Egyptian Rheumatol (2014) 36:131-7. doi: 10.1016/j.ejr.2013.12.006

114. Erdem F, Gündoğdu M, Kiki I, Ali Sari R, Kiziltunç A. Vascular Endothelial and Basic Fibroblast Growth Factor Serum Levels in Patients With Behçet's Disease. Rheumatol Int (2005) 25:599-603. doi: 10.1007/s00296-004-0484-7

115. Ganeb SS, Sabry HH, El-Assal MM, Kamal HM, Fayed AA, El-Shazly IM. Vascular Endothelial Growth Factor and Subclinical Atherosclerosis by Carotid Ultrasonography in Egyptian Patients With Behçet's Disease. Egyptian Rheumatol (2013) 35:87-94. doi: 10.1016/j.ejr.2012.12.001

116. Gheita TA, Sakr BR, Rabea RE, Abd ElHamid SM. Value of Hematological Indices Versus VEGF as Biomarkers of Activity in Behcet's Disease. Clin Rheumatol (2019) 38:2201-10. doi: 10.1007/s10067-019-04513-5

117. Ibrahim SE, Elshishtawy HF, Helmysamy A, Galal ZA. Role of Vascular Endothelial Growth Factor and Monocyte Chemoattractant Protein-1 in Behçet's Disease. Indian J Rheumatol (2011) 6:168-72. doi: 10.1016/S09733698(11)60202-1

118. Kul A, Ateş O, Alkan Melikoğlu M, Uğur M, Öztürk N, Erkayhan G, et al. Endocan Measurement for Active Behçet Disease. Arch Rheumatol (2017) 32:197-202. doi: 10.5606/ArchRheumatol.2017.6072
119. Ozdamar Y, Berker N, Bahar G, Soykan E, Bicer T, Ozkan SS, et al. Inflammatory Mediators and Posterior Segment Involvement in Ocular Behcet Disease. Eur J Ophthalmol (2009) 19:998-1003. doi: 10.1177/ 112067210901900616

120. Ozturk MA, Unverdi S, Oktar SO, Bukan N, Gulbahar O, Ureten K, et al. Vascular Endothelial Growth Factor and Carotid Intima-Media Thickness in Patients With Behcet's Disease. Clin Rheumatol (2008) 27:961-6. doi: 10.1007/s10067-007-0825-5

121. Sertoglu E, Omma A, Yucel C, Colak S, Sandikci SC, Ozgurtas T. The Relationship of Serum VEGF and sVEGFR-1 Levels With Vascular Involvement in Patients With Behcet's Disease. Scandinavian J Clin Lab Invest (2018) 78:443-9. doi: 10.1080/00365513.2018.1488179

122. Shaker O, Ay El-Deen MA, El Hadidi H, Grace BD, El Sherif H, Abdel Halim A. The Role of Heat Shock Protein 60, Vascular Endothelial Growth Factor and Antiphospholipid Antibodies in Behçet Disease. Br J Dermatol (2007) 156:32-7. doi: 10.1111/j.1365-2133.2006.07536.x

123. Yalcindag A, Gedik-Oguz Y, Yalcindag FN. The Relationship Between Serum Levels of Angiogenin, Bfgf, VEGF, and Ocular Involvement in Patients With Behcet's Disease. Graefes Arch Clin Exp Ophthalmol (2013) 251:1807-12. doi: 10.1007/s00417-013-2322-7

124. Breunis WB, Davila S, Shimizu C, Oharaseki T, Takahashi K, van Houdt M, et al. Disruption of Vascular Homeostasis in Patients With Kawasaki Disease: Involvement of Vascular Endothelial Growth Factor and Angiopoietins. Arthritis Rheumatism (2012) 64:306-15. doi: 10.1002/ art.33316

125. Hamamichi Y, Ichida F, Yu X, Hirono KI, Uese KI, Hashimoto I, et al. Neutrophils and Mononuclear Cells Express Vascular Endothelial Growth Factor in Acute Kawasaki Disease: its Possible Role in Progression of Coronary Artery Lesions. Pediatr Res (2001) 49:74-80. doi: 10.1203/ 00006450-200101000-00017

126. Maeno N, Takei S, Masuda K, Akaike H, Matsuo K, Kitajima I, et al. Increased Serum Levels of Vascular Endothelial Growth Factor in Kawasaki Disease. Pediatr Res (1998) 44:596-9. doi: 10.1203/00006450-19981000000021

127. Ohno T, Yuge T, Kariyazono H, Igarashi H, Joh-o K, Kinugawa N, et al. Serum Hepatocyte Growth Factor Combined With Vascular Endothelial Growth Factor as a Predictive Indicator for the Occurrence of Coronary Artery Lesions in Kawasaki Disease. Eur J Pediatr (2002) 161:105-11. doi: 10.1007/s00431-001-0860-1

128. Su Y, Feng SQ, Luo L, Liu RX, Yi QJ. Association Between IL-35 and Coronary Arterial Lesions in Children With Kawasaki Disease. Clin Exp Med (2019) 19:87-92. doi: 10.1007/s10238-018-0513-6

129. Ueno K, Nomura Y, Hashiguchi T, Masuda K, Morita Y, Hazeki D, et al. Platelet Vascular Endothelial Growth Factor is a Useful Predictor for Prognosis in Kawasaki Syndrome. Br J Haematol (2010) 148:285-92. doi: 10.1111/j.1365-2141.2009.07922.x

130. Zeng H, Wei R. Relationship Between Vascular Endothelial Growth Factor and Vascular Injury of Children Kawasaki Disease. Int J Rheumatic Dis (2016) 19:153. doi: 10.1111/1756-185X.12962

131. Akar S, Solmaz D, Bulbul H, Uslu S, Kozaci D, Karaca N, et al. Serum Level of the Vascular Endothelial Growth Factor is Elevated in Ankylosing Spondylitis and Osteocalcin may be Related With Osteoproliferation. Int $J$ Rheumatic Dis (2016) 19:239. doi: 10.1111/1756-185X.12962

132. Goldberger C, Dulak J, Duftner C, Weidinger F, Falkenbach A, Schirmer M. Vascular Endothelial Growth Factor (VEGF) in Ankylosing Spondylitis-a Pilot Study. Wien Med Wochenschr (2002) 152:223-5. doi: 10.1046/j.1563258x.2002.01118.x

133. Lin TT, Lu J, Qi CY, Yuan L, Li XL, Xia LP, et al. Elevated Serum Level of IL-27 and VEGF in Patients With Ankylosing Spondylitis and Associate With Disease Activity. Clin Exp Med (2015) 15:227-31. doi: 10.1007/s10238014-0281-x

134. Przepiera-Bedzak H, Fischer K, Brzosko M. EGF, Basic FGF, and Acidic FGF Levels and Their Association With Disease Activity and Extra-Articular Symptoms in Ankylosing Spondylitis. Polskie Archiwum Medycyny Wewnetrznej-Polish Arch Internal Med (2016) 126:290-2. doi: 10.20452/ pamw.3341

135. Przepiera-Bedzak H, Fischer K, Brzosko M. Serum IL-6 and IL-23 Levels and Their Correlation With Angiogenic Cytokines and Disease Activity in 
Ankylosing Spondylitis, Psoriatic Arthritis, and SAPHO Syndrome. Mediators Inflammation (2015) 2015:785705. doi: 10.1155/2015/785705

136. Przepiera-Bedzak H, Fischer K, Brzosko M. Serum Interleukin-18, Fetuin-a, Soluble Intercellular Adhesion Molecule-1, and Endothelin-1 in Ankylosing Spondylitis, Psoriatic Arthritis, and SAPHO Syndrome. Int J Mol Sci (2016) 17:1255. doi: 10.3390/ijms17081255

137. Sakellariou GT, Iliopoulos A, Konsta M, Kenanidis E, Potoupnis M, Tsiridis E, et al. Serum Levels of Dkk-1, Sclerostin and VEGF in Patients With Ankylosing Spondylitis and Their Association With Smoking, and Clinical, Inflammatory and Radiographic Parameters. Joint Bone Spine (2017) 84:309-15. doi: 10.1016/j.jbspin.2016.05.008

138. Solmaz D, Bulbul H, Uslu S, Kozaci LD, Karaca N, Akar S. Serum Level of The Vascular Endothelial Growth Factor is Elevated in Ankylosing Spondylitis And Osteocalcin May be Related Wth Osteoproliferation. Ann Rheumatic Dis (2015) 74:943-. doi: 10.1136/annrheumdis-2015-eular.4931

139. Solmaz D, Uslu S, Kozaci D, Karaca N, Bulbul H, Tarhan EF, et al. Evaluation of Periostin and Factors Associated With New Bone Formation in Ankylosing Spondylitis: Periostin may be Associated With the Wnt Pathway. Int J Rheumatic Dis (2018) 21:502-9. doi: 10.1111/1756$185 x .13186$

140. Torres L, Klingberg E, Nurkkala M, Carlsten H, Forsblad-d'Elia H. Hepatocyte Growth Factor is a Potential Biomarker for Osteoproliferation and Osteoporosis in Ankylosing Spondylitis. Osteoporosis Int (2019) 30:4419. doi: 10.1007/s00198-018-4721-4

141. Aksoy EK, Cetinkaya H, Savas B, Ensari A, Torgutalpv M, Efe C. Vascular Endothelial Growth Factor, Endostatin Levels and Clinical Features Among Patients With Ulcerative Colitis and Irritable Bowel Syndrome and Among Healthy Controls: A Cross-Sectional Analytical Study. Sao Paulo Med J (2018) 136:543-50. doi: 10.1590/1516-3180.2018.0274161118

142. Algaba A, Linares PM, Encarnacion Fernandez-Contreras M, Figuerola A, Calvet X, Guerra I, et al. The Effects of Infliximab or Adalimumab on Vascular Endothelial Growth Factor and Angiopoietin 1 Angiogenic Factor Levels in Inflammatory Bowel Disease: Serial Observations in 37 Patients. Inflammatory Bowel Dis (2014) 20:695-702. doi: 10.1097/mib. 0000000000000004

143. Di Sabatino A, Ciccocioppo R, Armellini E, Morera R, Ricevuti L, Cazzola P, et al. Serum bFGF and VEGF Correlate Respectively With Bowel Wall Thickness and Intramural Blood Flow in Crohn's Disease. Inflammation Bowel Dis (2004) 10:573-7. doi: 10.1097/00054725-200409000-00011

144. Dueñas Pousa I, Maté Jiménez J, Salcedo Mora X, Abreu MT, Moreno Otero R, Gisbert JP. Analysis of Soluble Angiogenic Factors in Crohn's Disease: A Preliminary Study. Gastroenterol Hepatol (2007) 30:518-24. doi: 10.1157/ 13111682

145. Ferrante M, Pierik M, Henckaerts L, Joossens M, Claes K, Van Schuerbeek N, et al. The Role of Vascular Endothelial Growth Factor (VEGF) in Inflammatory Bowel Disease. Inflammation Bowel Dis (2006) 12:870-8. doi: 10.1097/01.mib.0000235095.01608.10

146. Griga T, Gutzeit A, Sommerkamp C, May B. Increased Production of Vascular Endothelial Growth Factor by Peripheral Blood Mononuclear Cells in Patients With Inflammatory Bowel Disease. Eur J Gastroenterol Hepatol (1999) 11:175-9. doi: 10.1097/00042737-199902000-00019

147. Griga T, Tromm A, Spranger J, May B. Increased Serum Levels of Vascular Endothelial Growth Factor in Patients With Inflammatory Bowel Disease. Scand J Gastroenterol (1998) 33:504-8. doi: 10.1080/00365529850172070

148. Kanazawa S, Tsunoda T, Onuma E, Majima T, Kagiyama M, Kikuchi K. VEGF. basic-FGF, and TGF-beta in Crohn's Disease and Ulcerative Colitis: A Novel Mechanism of Chronic Intestinal Inflammation. Am J Gastroenterol (2001) 96:822-8. doi: 10.1111/j.1572-0241.2001.03527.x

149. Kapsoritakis A, Sfiridaki A, Maltezos E, Simopoulos K, Giatromanolaki A, Sivridis E, et al. Vascular Endothelial Growth Factor in Inflammatory Bowel Disease. Int J Colorectal Dis (2003) 18:418-22. doi: 10.1007/s00384-0030495-y

150. Kleiner G, Zanin V, Monasta L, Crovella S, Caruso L, Milani D, et al. Pediatric Patients With Inflammatory Bowel Disease Exhibit Increased Serum Levels of Proinflammatory Cytokines and Chemokines, But Decreased Circulating Levels of Macrophage Inhibitory Protein-1 $\beta$, Interleukin-2 and Interleukin-17. Exp Ther Med (2015) 9:2047-52. doi: $10.3892 /$ etm. 2015.2370
151. Magro F, Araujo F, Pereira P, Meireles E, Diniz-Ribeiro M, Velosom FT. Soluble Selectins, sICAM, sVCAM, and Angiogenic Proteins in Different Activity Groups of Patients With Inflammatory Bowel Disease. Dig Dis Sci (2004) 49:1265-74. doi: 10.1023/b:ddas.0000037822.55717.31

152. Pousa ID, Algaba A, Linares PM, Sanz-Cameno P, Mate J, Moreno-Otero R, et al. Corticosteroids Modulate Angiogenic Soluble Factors in Ulcerative Colitis Patients. Digestive Dis Sci (2011) 56:871-9. doi: 10.1007/s10620-010$1327-\mathrm{x}$

153. Pousa ID, Mate J, Salcedo-Mora X, Abreu MT, Moreno-Otero R, Gisbert JP. Role of Vascular Endothelial Growth Factor and Angiopoietin Systems in Serum of Crohn's Disease Patients. Inflammatory Bowel Dis (2008) 14:61-7. doi: 10.1002/ibd.20269

154. Schurer-Maly CC, Fried M, Maly FE, Binek J, Frenzer A. Vascular Endothelial Growth Factor in Serum of Patients With Inflammatory Bowel Disease [1]. Scandinavian J Gastroenterol (1997) 32:959-60. doi: 10.3109/00365529709011209

155. deZoeten EF, Battista KD, Colson SB, Lovell MA, Kessler BE, Isfort RW, et al. Markers of Hypoxia Correlate With Histologic and Endoscopic Severity of Colitis in Inflammatory Bowel Disease. Hypoxia (Auckl) (2020) 8:1-12. doi: $10.2147 / \mathrm{hp} . S 219049$

156. Wiercinska-Drapalo A, Jaroszewicz J, Parfieniuk A, Lapinski TW, Rogalska M, Prokopowicz D. Pigment Epithelium-Derived Factor in Ulcerative Colitis: Possible Relationship With Disease Activity. Regul Peptides (2007) 140:1-4. doi: 10.1016/j.regpep.2006.11.006

157. Akman A, Dicle O, Yilmaz F, Coskun M, Yilmaz E. Discrepant Levels of Vascular Endothelial Growth Factor in Psoriasis Patients Treated With PUVA, Re-PUVA and Narrow-Band UVB. Photodermatol Photoimmunol Photomed (2008) 24:123-7. doi: 10.1111/j.1600-0781.2008.00349.x

158. Anderson KS, Petersson S, Wong J, Shubbar E, Lokko NN, Carlstrom M, et al. Elevation of Serum Epidermal Growth Factor and Interleukin 1 Receptor Antagonist in Active Psoriasis Vulgaris. Br J Dermatol (2010) 163:1085-9. doi: 10.1111/j.1365-2133.2010.09990.x

159. Batycka-Baran A, Paprocka M, Baran W, Szepietowski JC. Decreased Number of Circulating Endothelial Progenitor Cells (CD133+/KDR+) in Patients With Psoriatic Arthritis. Acta Dermato-Venereol (2016) 96:754-7. doi: 10.2340/00015555-2353

160. Batycka-Baran A, Paprocka M, Krawczenko A, Kantor A, Dus D, Szepietowski JC. Reduced Number of Circulating Endothelial Progenitor Cells $(\mathrm{CD} 133+/ \mathrm{KDR}+)$ in Patients With Plaque Psoriasis. Dermatology (2012) 225:88-92. doi: 10.1159/000341534

161. Capkin AA, Demir S, Mentese A, Bulut C, Ayar A. Can Signal peptide-CUBEGF Domain-Containing Protein (SCUBE) Levels be a Marker of Angiogenesis in Patients With Psoriasis? Arch Dermatol Res (2017) 309:203-7. doi: 10.1007/s00403-017-1722-7

162. Bhushan M, McLaughlin B, Weiss JB, Griffiths CE. Levels of Endothelial Cell Stimulating Angiogenesis Factor and Vascular Endothelial Growth Factor are Elevated in Psoriasis. Br J Dermatol (1999) 141:1054-60. doi: 10.1046/ j.1365-2133.1999.03205.x

163. Creamer D, Allen M, Jaggar R, Stevens R, Bicknell R, Barker J. Mediation of Systemic Vascular Hyperpermeability in Severe Psoriasis by Circulating Vascular Endothelial Growth Factor. Arch Dermatol (2002) 138:791-6. doi: 10.1001/archderm.138.6.791

164. Flisiak I, Zaniewski P, Rogalska M, Mysliwiec H, Jaroszewicz J, Chodynicka B. Effect of Psoriasis Activity on VEGF and its Soluble Receptors Concentrations in Serum and Plaque Scales. Cytokine (2010) 52:225-9. doi: $10.1016 /$ j.cyto.2010.09.012

165. Fink AM, Cauza E, Hassfeld W, Dunky A, Bayer PM, Jurecka W, et al. Vascular Endothelial Growth Factor in Patients With Psoriatic Arthritis. Clin Exp Rheumatol (2007) 25:305-8.

166. Kaur S, Zilmer K, Leping V, Zilmer M. Comparative Study of Systemic Inflammatory Responses in Psoriasis Vulgaris and Mild to Moderate Allergic Contact Dermatitis. Dermatology (2012) 225:54-61. doi: 10.1159/000339866

167. Meki AR, Al-Shobaili H. Serum Vascular Endothelial Growth Factor, Transforming Growth Factor $\beta 1$, and Nitric Oxide Levels in Patients With Psoriasis Vulgaris: Their Correlation to Disease Severity. J Clin Lab Anal (2014) 28:496-501. doi: 10.1002/jcla.21717

168. Midde HS, Priyadarssini M, Rajappa M, Munisamy M, Raj PSM, Singh S, et al. Interleukin-9 Serves as a Key Link Between Systemic Inflammation and 
Angiogenesis in Psoriasis. Clin Exp Dermatol. (2021) 46(1):50-7. doi: $10.1111 /$ ced.14335

169. Nielsen HJ, Christensen IJ, Svendsen MN, Hansen U, Werther K, Brünner N, et al. Elevated Plasma Levels of Vascular Endothelial Growth Factor and Plasminogen Activator Inhibitor-1 Decrease During Improvement of Psoriasis. Inflammation Res (2002) 51:563-7. doi: 10.1007/pl00012428

170. Nofal A, Al-Makhzangy I, Attwa E, Nassar A, Abdalmoati A. Vascular Endothelial Growth Factor in Psoriasis: An Indicator of Disease Severity and Control. J Eur Acad Dermatol Venereol (2009) 23:803-6. doi: 10.1111/j.14683083.2009.03181.x

171. Przepiera-Bedzak H, Fischer K, Brzosko M. Serum Levels of Angiogenic Cytokines in Psoriatic Arthritis and SAPHO Syndrome. Polskie Archiwum Medycyny Wewnetrznej-Polish Arch Internal Med (2013) 123:297-302. doi: 10.20452/pamw.1772

172. Shahidi-Dadras M, Haghighatkhah HR, Abdollahimajd F, Younespour S, Kia MP, Zargari O. Correlation Between Vascular Endothelial Growth Factor and Subclinical Atherosclerosis in Patients With Psoriasis. Int J Dermatol (2016) 55:52-9. doi: 10.1111/ijd.12842

173. Shahidi-Dadras M, Abdollahimajd F, Younespour S, Nikvar M. Serum Vascular Endothelial Growth Factor in Iranian Patients With ModerateSevere Psoriasis Before and After Treatment: A Pasi-75 Response as a Practical Treatment Goal. Iranian J Dermatol (2016) 19:119-24.

174. Takahashi H, Tsuji H, Hashimoto Y, Ishida-Yamamoto A, Iizuka H. Serum Cytokines and Growth Factor Levels in Japanese Patients With Psoriasis. Clin Exp Dermatol (2010) 35:645-9. doi: 10.1111/j.1365-2230.2009.03704.x

175. Zheng YZ, Chen CF, Jia LY, Yu TG, Sun J, Wang XY. Correlation Between microRNA-143 in Peripheral Blood Mononuclear Cells and Disease Severity in Patients With Psoriasis Vulgaris. Oncotarget (2017) 8:51288-95. doi: 10.18632 /oncotarget. 17260

176. Figueroa-Vega N, Sanz-Cameno P, Moreno-Otero R, Sanchez-Madrid F, Gonzalez-Amaro R, Marazuela M. Serum Levels of Angiogenic Molecules in Autoimmune Thyroid Diseases and Their Correlation With Laboratory and Clinical Features. J Clin Endocrinol Metab (2009) 94:1145-53. doi: 10.1210/ jc.2008-1571

177. Iitaka M, Miura S, Yamanaka K, Kawasaki S, Kitahama S, Kawakami Y, et al. Increased Serum Vascular Endothelial Growth Factor Levels and Intrathyroidal Vascular Area in Patients With Graves' Disease and Hashimoto's Thyroiditis. J Clin Endocrinol Metab (1998) 83:3908-12. doi: 10.1210/jcem.83.11.5281

178. Kajdaniuk D, Marek B, Niedziolka-Zielonka D, Foltyn W, Nowak M, Sieminska L, et al. Transforming Growth Factor Beta 1 (TGF Beta 1) and Vascular Endothelial Growth Factor (VEGF) in the Blood of Healthy People and Patients With Graves' Orbitopathy - a New Mechanism of Glucocorticoids Action? Endokrynol Polska (2014) 65:348-56. doi: 10.5603/ ep.2014.0048

179. Rancier M, Zaaber I, Stathopoulou MG, Chatelin J, Saleh A, Marmouch H, et al. Pro- and Anti-Angiogenic VEGF mRNAs in Autoimmune Thyroid Diseases. Autoimmunity (2016) 49:366-72. doi: 10.1080/08916934. 2016.1199019

180. Ye X, Liu J, Wang Y, Bin L, Wang J. Increased Serum VEGF and b-FGF in Graves' Ophthalmopathy. Graefe's Arch Clin Exp Ophthalmol (2014) 252:1639-44. doi: 10.1007/s00417-014-2662-y

181. Breunis WB, Biezeveld MH, Geissler J, Ottenkamp J, Kuipers IM, Lam J, et al. Vascular Endothelial Growth Factor Gene Haplotypes in Kawasaki Disease. Arthritis Rheumatol (2006) 54:1588-94. doi: 10.1002/art.21811

182. Leung DW, Cachianes G, Kuang WJ, Goeddel DV, Ferrara N. Vascular Endothelial Growth Factor is a Secreted Angiogenic Mitogen. Science (1989) 246:1306-9. doi: 10.1126/science.2479986

183. Danve A, O'Dell J. The Ongoing Quest for Biomarkers in Ankylosing Spondylitis. Int J Rheum Dis (2015) 18:826-34. doi: 10.1111/1756$185 x .12779$

184. Huang SM, Liao WT, Lin CF, Sun HS, Chow NH. Effectiveness and Mechanism of Preoperative Lugol Solution for Reducing Thyroid Blood Flow in Patients With Euthyroid Graves' Disease. World J Surgery (2016) 40:505-9. doi: 10.1007/s00268-015-3298-8

185. Berrahmoune H, Lamont JV, Herbeth B, FitzGerald PS, Visvikis-Siest S. Biological Determinants of and Reference Values for Plasma interleukin-8, Monocyte Chemoattractant protein-1, Epidermal Growth Factor, and Vascular Endothelial Growth Factor: Results From the STANISLAS Cohort. Clin Chem (2006) 52:504-10. doi: 10.1373/clinchem.2005.055798

Conflict of Interest: The authors declare that the research was conducted in the absence of any commercial or financial relationships that could be construed as a potential conflict of interest.

Copyright $\odot 2021$ Zhan, Li, Liu, Cheng, Yan and Li. This is an open-access article distributed under the terms of the Creative Commons Attribution License (CC BY). The use, distribution or reproduction in other forums is permitted, provided the original author(s) and the copyright owner(s) are credited and that the original publication in this journal is cited, in accordance with accepted academic practice. No use, distribution or reproduction is permitted which does not comply with these terms. 